\title{
Effect of Salting Duration on Lipid Oxidation and the Fatty Acid Content of Dry-Cured Lacón
}

\author{
Rubén Garrido ${ }^{1}$, José M. Lorenzo ${ }^{1}$, Inmaculada Franco ${ }^{1} \&$ Javier Carballo $^{1}$ \\ ${ }^{1}$ Área de Tecnología de los Alimentos, Facultad de Ciencias de Orense, Universidad de Vigo, Spain \\ Correspondence: Javier Carballo, Área de Tecnología de los Alimentos, Facultad de Ciencias de Orense, \\ Universidad de Vigo, Spain. Tel: 34-988-387052. E-mail: carbatec@uvigo.es
}

Received: September 20, 2013 Accepted: November 25, 2013 Online Published: December 16, 2013

doi:10.5539/jfr.v3n1p46 URL: http://dx.doi.org/10.5539/jfr.v3n1p46

\begin{abstract}
We investigated the effects of salting duration (3, 4 or 5 days) on lipid oxidation and the total fatty acid content of muscular fat and subcutaneous fat during the manufacturing of dry-cured lacón, a traditional meat product made in NW Spain from pork foreleg. Two batches of lacón were processed using each salting duration. In each batch, samples were analysed at seven different times throughout the manufacturing process. In each sample, the moisture and $\mathrm{NaCl}$ contents, and the peroxide value of the fat and the total fatty acid contents were determined in both the muscular and the subcutaneous fat.

Increasing the salting duration significantly increased lipid oxidation (as indicated by peroxide values), in both the muscular and the subcutaneous fat and at all sampling times throughout the manufacturing process. At the end of the ripening stage, the average peroxide values were 7.69, 17.79 and 21.72 meq. of $\mathrm{O}_{2} / \mathrm{kg}$ of subcutaneous fat and $10.78,24.96$ and 26.48 meq. of $\mathrm{O}_{2} / \mathrm{kg}$ of muscular fat, in the batches salted for 3,4 and 5 days, respectively.

Salting duration significantly affected the fatty acid content, particularly that of polyunsaturated fatty acids and the linoleic acid within these. The polyunsaturated fatty acid content of lacón pieces salted for 3 days were significantly higher than those of pieces salted for 4 or 5 days, in both the muscular and subcutaneous fat.
\end{abstract}

Keywords: fat, lacón, fatty acid, oxidation, peroxide, salting

\section{Introduction}

Dry-cured lacón is a salted, dried and ripened meat product manufactured in the northwest of Spain by traditional methods that use pork foreleg as the raw material. The manufacturing process is very similar to that of dry-cured hams, in regard to the steps, equipment and facilities required (Marra et al., 1999). The final product is usually consumed after cooking; however, it can be consumed in the raw state if the ripening period has been long enough.

This product is highly appreciated and consumed abundantly in NW Spain, with good marketing implementation. However, the quality of manufactured lacón is very variable (Lorenzo et al., 2002), which can spoil the image of the product and hamper its promotion. The variable quality is mainly the result of insufficient standardization of the manufacturing process. It is also due to the limited knowledge about the effects of different factors on the biochemical and microbiological phenomena that occur throughout the manufacturing process and that are responsible for the organoleptic characteristics and quality attributes of the final product.

Several studies have been carried out in recent years to investigate the microbiological (Vilar et al., 2000) and biochemical (Lorenzo et al., 2003) characteristics of lacón at different stages of the manufacturing process. The effects of the use of several additives on the microbiological and chemical phenomena that occur during the process have been also studied more recently (Lorenzo et al., 2007; 2008; 2010; 2012). However, the effects of other aspects of the manufacturing process on the characteristics and attributes of the final product remain unknown.

The organoleptic and nutritional characteristics of raw-cured meat products are the result of the physicochemical and chemical changes that occur during manufacture, mainly dehydration, proteolysis, lipolysis and autooxidation (Shahidi et al., 1986; Toldrá et al., 1997; Toldrá \& Flores, 1998; Gandemer, 2002; Toldrá \& Flores, 2007). The effect of the level of salt on the activity of tissue proteases and lipases involved in these changes has 
been widely demonstrated (Sárraga et al., 1989; Rico et al., 1990; Motilva \& Toldrá, 1993). The effect of the $\mathrm{NaCl}$ concentrations on lipid oxidation in specific cured meat products has also been described (Coutron-Gambotti et al., 1999). According to this, the duration of salting and the resulting salt content in the meat pieces should affect the proteolytic, lipolytic and oxidative phenomena that occur during ripening as well as the organoleptic and nutritional attributes of the final product.

Following the guidelines established for the manufacture of cured hams, the recommended duration of salting is one day per kg of weight of the raw piece (Marra et al., 1999). However, given the particular characteristics of pork shoulder (larger surface per unit of weight and less thickness than hams), this may not be the most appropriate salting duration for dry-cured lacón.

The effects of the salting duration on the proteolytic processes during the manufacture of dry-cured lacón and on the sensorial characteristics of the final product have been investigated in a previous study (Garrido et al., 2012). The aim of the present study was to assess the effect of the salting duration on lipid oxidation and the fatty acid content of the pieces during processing. The overall conclusions of these studies will help manufacturers to standardize production in relation to the salting process, thus yielding a more homogeneous product with improved organoleptic and nutritional qualities.

\section{Materials and Methods}

\subsection{Samples}

Six batches of lacón were manufactured following the usual industrial methods, as described by Garrido et al. (2012). Each batch consisted of nine lacón pieces that at the green stage weighed $4 \mathrm{~kg}$ each. Two batches were salted for 3 days $(0.75$ days $/ \mathrm{kg}$ of the raw piece), two batches for 4 days $(1 \mathrm{day} / \mathrm{kg})$ and two batches for 5 days $(1.25$ days $/ \mathrm{kg})$.

In each batch of lacón, samples were obtained from fresh pieces, at the end of the salting stage, after 7 and 14 days post-salting, and after 7, 14, 28, 56, and 84 days of drying-ripening. Each sample comprised one whole piece. Lacón pieces were transported to the laboratory refrigerated $\left(<4^{\circ} \mathrm{C}\right)$ and they were analysed on arrival. In the laboratory, the pieces were skinned with the aid of a knife, and the subcutaneous fat was taken. The pieces were then boned and the muscular portion was obtained. Both the subcutaneous fat and the muscular portion were triturated separately in a high-capacity mincing machine.

\subsection{Analytical Methods}

The moisture and $\mathrm{NaCl}$ contents were assessed in duplicate in each sample (piece), following the procedures reported by Marra et al. (1999). The muscular and subcutaneous fat was extracted following the procedure described by Folch et al. (1957). The peroxide values of the fat were determined following the Spanish Official Standard UNE 55.011 (B.O.E., 1977).

Fat extracts were methylated and fatty acid profiles of both muscular and subcutaneous lipids were determined following the procedure described by Franco et al. (2006).

Fatty acid methyl esters were analysed by Gas Chromatography in a chromatograph (Thermo Finnigan Trace GC, Thermo Finnigan, Austin, Texas, USA), equipped with a Split/Splitless AI 3000 auto-injector and a flame ionization detector (FID). The different fatty acids were separated on an Innowax column: $30 \mathrm{~m} ; 0.25 \mathrm{~mm}$ ID; $0.25 \mu \mathrm{m}$ film thickness (Agilent Technologies, Palo Alto, CA, USA). The temperature of the detector was $250{ }^{\circ} \mathrm{C}$ and that of the injector $230^{\circ} \mathrm{C}$. The chromatographic conditions used in the fatty acid determination were as follows: initial oven temperature: $50^{\circ} \mathrm{C}$ for 1 minute; ramp1: $5^{\circ} \mathrm{C} / \mathrm{min}$ to $248{ }^{\circ} \mathrm{C}$; ramp2: $248{ }^{\circ} \mathrm{C}$ for 6 minutes. The gasses used were air $(350 \mathrm{~mL} / \mathrm{min})$, hydrogen $(335 \mathrm{~mL} / \mathrm{min})$ and helium (carrier gas) $(30 \mathrm{~mL} / \mathrm{min})$.

For comparative purposes, a standard containing between $2 \%$ and $4 \%$ of each of the following fatty acids was obtained from Sigma-Aldrich Chemical Co. (St Louis, MO, USA): butyric (C4); caproic (C6), caprilic (C8); capric (C10); undecanoic (C11); lauric (C12); tridecanoic (C13); myristic (C14); myristoleic (C14:1); pentadecanoic (C15); cis-10 pentadecanoic (C15:1); palmitic (C16); palmitoleic (C16:1); margaric (17); cis-10 heptadecanoic (C17:1); stearic (C18); oleic (C18:1 cis); elaidic (C18:1 trans); linoleic (C18:2); linoelaidic (C18:2 trans); linolenic (C18:3); arachidic (C20); cis-11 eicosanoic (C20:1); cis-11, 14 eicosadienoic (C20:2); cis-11, 14, 17 eicosatrienoic (C20:3); arachidonic (C20:4); heneicosanoic (C21); behenic (C22); erucic (C22:1); cis-13, 16 docosadienoic (C22:2); cis-4, 7, 10, 13, 16, 19 docosahexanoic (C22:6); tricosaenoic (C23); lignoceric (C24); and nervonic (C24:1).

All the samples and standards (Sigma-Aldrich) were injected onto the chromatography column in duplicate (at least). Tests of repeatability were performed by injecting a standard and a sample consecutively six times a day. 
Tests of reproducibility were also carried out, by injecting the standard and the sample twice a day for 3 days under the same experimental conditions. There were no significant differences $(P<0.05)$ between the results obtained in either of the tests.

The fatty acids were quantified by using an internal standard (C13 and C19 fatty acids), and calibration curves were prepared for this purpose, by using different concentrations of each methyl ester. Fatty acids were determined in duplicate (at least) in each sample. Results for each fatty acid are expressed as percentages of the total fatty acid composition.

\subsection{Statistical Analysis}

Analysis of variance (ANOVA) was used to evaluate the effect of the ripening time and the effect of the salting duration for each ripening time, and any difference were considered significant at $P<0.05$. When differences were indicated, mean values were compared by the least squares difference (LSD) test, with the aid of Statistica 5.1 for Windows (Statsoft Inc, 1996, Tulsa, OK, USA).

\section{Results and Discussion}

Table 1 shows the changes in the total solid (TS) and $\mathrm{NaCl}$ contents in the muscular portion of the lacón pieces during manufacture in relation to the different salting periods. The total solid content increased gradually and significantly $(\mathrm{P}<0.05)$ throughout the process, and the final values reached were $47.86 \pm 3.64,48.31 \pm 0.67$ and $50.16 \pm 1.54 \mathrm{~g} / 100 \mathrm{~g}$ in the pieces of the batches salted for 3,4 and 5 days, respectively.

Table 1. Evolution of the total solid $(\mathrm{g} / 100 \mathrm{~g})$ and $\mathrm{NaCl}(\mathrm{g} / 100 \mathrm{~g}$ of total solids) contents during the manufacture of dry-cured lacón salted along the different times (mean \pm standard deviation values of two batches)

\begin{tabular}{|c|c|c|c|c|c|c|c|c|c|c|}
\hline & & \multirow{2}{*}{ Fresh piece } & \multirow{2}{*}{$\begin{array}{l}\text { After } \\
\text { salting }\end{array}$} & \multicolumn{2}{|c|}{ Post-salting (days) } & \multicolumn{5}{|c|}{ Drying-ripening (days) } \\
\hline & & & & 7 & 14 & 7 & 14 & 28 & 56 & 84 \\
\hline \multirow{2}{*}{$\begin{array}{c}\text { Salting along } 3 \\
\text { days }\end{array}$} & $\begin{array}{l}\text { Total } \\
\text { solids }\end{array}$ & $25.36 \pm 0.71^{\mathrm{al}}$ & $32.25 \pm 1.47^{\mathrm{b} 1}$ & $37.43 \pm 0.98^{\mathrm{bcl}}$ & $37.64 \pm 1.68^{\mathrm{bc} 1}$ & $37.35 \pm 0.11^{\mathrm{bc} 1}$ & $39.46 \pm 2.55^{\mathrm{c} 1}$ & $42.84 \pm 0.02^{\mathrm{cd} 1}$ & $44.11 \pm 1.66^{\mathrm{de} 1}$ & $47.86 \pm 3.64^{\mathrm{el}}$ \\
\hline & $\mathrm{NaCl}$ & $0.39 \pm 0.07^{\mathrm{a} 1}$ & $4.58 \pm 0.36^{\mathrm{b} 1}$ & $5.87 \pm 0.23^{\mathrm{bcl}}$ & $7.50 \pm 1.82^{\mathrm{cd} 1}$ & $9.39 \pm 0.03^{\mathrm{d} 1}$ & $9.65 \pm 0.10^{\mathrm{de} 1}$ & $10.37 \pm 0.18^{\text {de } 1}$ & $10.90 \pm 0.46^{\mathrm{de} 1}$ & $11.23 \pm 0.08^{\mathrm{e} 1}$ \\
\hline \multirow{2}{*}{$\begin{array}{c}\text { Salting along } 4 \\
\text { days }\end{array}$} & $\begin{array}{l}\text { Total } \\
\text { solids }\end{array}$ & $25.86 \pm 0.93^{\mathrm{a} 1}$ & $31.41 \pm 2.34^{\mathrm{b} 1}$ & $33.69 \pm 0.57^{\mathrm{bc} 1}$ & $36.26 \pm 1.41^{\mathrm{cl}}$ & $37.32 \pm 0.28^{\mathrm{cd} 1}$ & $40.65 \pm 1.66^{\mathrm{de} 1}$ & $42.95 \pm 1.56^{\mathrm{e} 1}$ & $44.03 \pm 0.91^{\mathrm{ef} 1}$ & $48.31 \pm 0.67^{\mathrm{ef} 1}$ \\
\hline & $\mathrm{NaCl}$ & $0.42 \pm 0.03^{\mathrm{a} 1}$ & $5.17 \pm 0.06^{\mathrm{b} 1}$ & $7.21 \pm 0.71^{\mathrm{c} 1}$ & $8.62 \pm 0.57^{\mathrm{cl}}$ & $10.48 \pm 1.03^{\mathrm{df} 1}$ & $10.32 \pm 0.09^{\mathrm{de} 1}$ & $11.09 \pm 0.19^{\text {ef } 1}$ & $11.45 \pm 0.01^{\mathrm{ef} 1}$ & $12.22 \pm 0.75^{\mathrm{f} 1}$ \\
\hline \multirow{2}{*}{$\begin{array}{c}\text { Salting along } 5 \\
\text { days }\end{array}$} & $\begin{array}{l}\text { Total } \\
\text { solids }\end{array}$ & $24.97 \pm 3.10^{\mathrm{a} 1}$ & $31.62 \pm 0.63^{\mathrm{b} 1}$ & $35.50 \pm 0.85^{\text {bc1 }}$ & $36.87 \pm 3.62^{\mathrm{cl}}$ & $41.52 \pm 0.50^{\mathrm{d} 1}$ & $38.64 \pm 3.58^{\text {dce } 1}$ & $40.81 \pm 0.01^{\mathrm{e} 1}$ & $46.85 \pm 4.05^{\mathrm{fl}}$ & $50.16 \pm 1.54^{\mathrm{fl}}$ \\
\hline & $\mathrm{NaCl}$ & $0.47 \pm 0.04^{\mathrm{a} 1}$ & $5.80 \pm 0.55^{\mathrm{b} 1}$ & $9.67 \pm 0.64^{\mathrm{c} 2}$ & $10.40 \pm 1.04^{\mathrm{cd} 1}$ & $10.85 \pm 1.03^{\text {cde } 1}$ & $11.51 \pm 0.25^{\text {def } 1}$ & $12.39 \pm 1.83^{\mathrm{efl}}$ & $12.42 \pm 1.26^{\mathrm{efl}}$ & $12.75 \pm 0.73^{\mathrm{fl}}$ \\
\hline
\end{tabular}

\footnotetext{
$\overline{\mathrm{a}-\mathrm{f}}$ Mean values in the same row (corresponding to the same parameter) not followed by a common letter differ significantly $(\mathrm{P}<0.05)$.

${ }^{1-2-3}$ Mean values corresponding to the same parameter and sampling time not followed by a common number differ significantly $(\mathrm{P}<0.05)$ between the batches salted along 3,4 and 5 days.
}

Although the manufacturing time is shorter for lacón ( 3 months) than for other raw-cured meat products made from whole pieces, such as hams (usually between 6 and 12 months), the final total solid contents in the present study are similar to or even higher than those reported for hams (Astiasarán et al., 1991; Monin et al., 1996; Bellatti \& Reverbi, 1997; Schivazappa et al., 1998; Bañón et al., 1999; Toscani et al., 2000). This may be explained by the fact that pork foreleg pieces are smaller and thinner than hams, and the surface area per unit of weight is therefore larger. This favours dehydration throughout the post-salting and drying-ripening stages, and therefore the weight losses in lacón are even higher than those observed in hams (Lorenzo et al., 2005). This may also cause greater loss of moisture due to osmotic phenomena during the salting process.

An increase in the total solid contents was observed after salting, and the increase was significant $(\mathrm{P}<0.05)$ in the pieces salted for 4 and 5 days.

The loss of moisture was more marked during the drying-ripening stage, as a result of the longer duration of this period and the environmental conditions (higher temperature and lower relative humidity than in the other 
stages). The total solid contents were higher in the pieces that were salted for longer, although the differences were not statistically significant.

The mean values of total solid contents at the end of the manufacturing process were similar to those reported by Marra et al. (1999) and slightly lower than those reported by Lorenzo et al. $(2003 ; 2008)$ for the same meat product.

The $\mathrm{NaCl}$ contents increased significantly $(\mathrm{P}<0.05)$ during salting, reaching average values ranging from 4.58 $\mathrm{g} / 100 \mathrm{~g}$ of T.S. (in the batches salted for 3 days) to $5.80 \mathrm{~g} / 100 \mathrm{~g}$ of T.S. (in the batches salted for 5 days). The increase was even higher during the post salting stage; at the end of this stage, the average $\mathrm{NaCl}$ contents were $7.50,8.62$ and $10.40 \mathrm{~g} \mathrm{NaCl} / 100 \mathrm{~g}$ of T.S. in the batches salted for 3, 4 and 5 days, respectively. During the drying-ripening stage, a slight increase was observed, this being only significant $(\mathrm{P}<0.05)$ during the first 7 days of this stage and in the batches salted for 4 and 5 days. The mean $\mathrm{NaCl}$ contents at the end of the manufacturing process were $11.23,12.22$ and $12.75 \mathrm{~g} \mathrm{NaCl} / 100 \mathrm{~g}$ of TS in the batches salted for 3,4 and 5 days, respectively. Although the salt contents of the pieces salted for the three times were different, especially at the post-salting and drying-ripening stages, the differences were not significant $(\mathrm{P}>0.05)$.

The $\mathrm{NaCl}$ contents of the final product were generally lower than those reported by other authors for this meat product (Marra et al., 1999; Lorenzo et al., 2008). According to previous reported data in the relevant literature, the $\mathrm{NaCl}$ contents in lacón pieces at the end of the manufacturing process in this study are at the lower end of the range of values described for different varieties of ham (Astiasarán et al., 1988; 1991; Buscailhon et al., 1994a; Gou et al., 1995; Bellatti \& Reverbi, 1997; Monin et al., 1997; Martín et al., 1998; Schivazappa et al., 1998; Toscani et al., 2000; Candek-Potokar et al., 2002; Moller et al., 2003; Mariscal et al., 2004).

Table 2 shows the variation in the peroxide values in both the muscular and subcutaneous fat of the pieces during manufacture of the batches salted for different times.

Table 2. Evolution of the peroxide values (meq $\mathrm{O}_{2} / \mathrm{kg}$ of fat) in the muscular and subcutaneous fat during the manufacture of dry-cured lacón salted along the different times (mean \pm standard deviation values of two batches)

\begin{tabular}{|c|c|c|c|c|c|c|c|c|c|c|}
\hline & & \multirow{2}{*}{$\begin{array}{l}\text { Fresh } \\
\text { piece }\end{array}$} & \multirow{2}{*}{ After salting } & \multicolumn{2}{|c|}{ Post-salting (days) } & \multicolumn{5}{|c|}{ Drying-ripening (days) } \\
\hline & & & & 7 & 14 & 7 & 14 & 28 & 56 & 84 \\
\hline \multirow{2}{*}{ Salting along 3 days } & Muscular fat & $2.38 \pm 0.13^{\mathrm{a} 1}$ & $5.56 \pm 0.63^{\mathrm{bl}}$ & $6.12 \pm 0.20^{\mathrm{bl}}$ & $4.71 \pm 0.27^{\mathrm{cl}}$ & $4.56 \pm 0.34^{\mathrm{cl}}$ & $3.44 \pm 0.51^{\mathrm{a} 1}$ & $8.23 \pm 0.95^{\mathrm{d} 1}$ & $10.34 \pm 0.63^{\mathrm{el}}$ & $10.78 \pm 0.10^{\mathrm{el}}$ \\
\hline & Subcutaneous fat & $2.56 \pm 0.16^{\mathrm{al}}$ & $4.29 \pm 0.38^{\mathrm{bl}}$ & $2.29 \pm 0.35^{\mathrm{al}}$ & $2.86 \pm 0.74^{\mathrm{al}}$ & $2.21 \pm 0.32^{\mathrm{al}}$ & $3.52 \pm 0.28^{\mathrm{cl}}$ & $6.04 \pm 0.08^{\mathrm{d} 1}$ & $9.38 \pm 0.41^{\mathrm{el}}$ & $7.68 \pm 0.07^{\mathrm{el}}$ \\
\hline \multirow{2}{*}{ Salting along 4 days } & Muscular fat & $3.32 \pm 0.91^{\mathrm{al}}$ & $8.39 \pm 2.14^{\mathrm{b} 2}$ & $9.80 \pm 1.16^{\mathrm{b} 2}$ & $10.98 \pm 1.42^{\mathrm{b} 2}$ & $9.88 \pm 0.10^{\mathrm{b} 2}$ & $6.97 \pm 1.37^{\mathrm{c} 2}$ & $17.83 \pm 2.94^{\mathrm{d} 2}$ & $20.83 \pm 1.57^{\mathrm{d} 2}$ & $24.96 \pm 0.00^{\mathrm{e}}$ \\
\hline & Subcutaneous fat & $3.46 \pm 0.73^{\mathrm{al}}$ & $4.95 \pm 0.05^{\mathrm{bl}}$ & $5.92 \pm 0.04^{\mathrm{c} 2}$ & $5.95 \pm 1.37^{\mathrm{c} 2}$ & $6.91 \pm 1.42^{\mathrm{d} 2}$ & $8.46 \pm 0.75^{\mathrm{d} 2}$ & $8.91 \pm 1.45^{\mathrm{d} 2}$ & $14.81 \pm 1.27^{e 2}$ & $17.79 \pm 0.11^{\mathrm{e} 2}$ \\
\hline \multirow{2}{*}{ Salting along 5 days } & Muscular fat & $3.49 \pm 0.70^{\mathrm{al}}$ & $10.14 \pm 2.20^{\mathrm{b} 2}$ & $14.05 \pm 0.63^{\mathrm{c} 3}$ & $15.81 \pm 2.83^{\mathrm{c} 3}$ & $20.06 \pm 1.06^{\mathrm{d} 3}$ & $21.81 \pm 5.56^{\mathrm{d} 3}$ & $24.71 \pm 1.11^{\mathrm{d} 3}$ & $22.58 \pm 4.58^{\mathrm{d} 2}$ & $26.48 \pm 6.05^{\mathrm{d} 3}$ \\
\hline & Subcutaneous fat & $2.94 \pm 1.40^{\mathrm{al}}$ & $5.86 \pm 2.82^{\mathrm{bl}}$ & $6.90 \pm 1.40^{\mathrm{c} 2}$ & $5.93 \pm 0.01^{\mathrm{b} 2}$ & $11.33 \pm 0.67^{\mathrm{d} 3}$ & $13.83 \pm 0.01^{\mathrm{e} 3}$ & $15.76 \pm 0.09^{\mathrm{f3}}$ & $17.43 \pm 0.62^{g^{3}}$ & $21.72 \pm 2.76^{h 3}$ \\
\hline
\end{tabular}

${ }^{\mathrm{a}}$ - $\mathrm{h}$ Mean values in the same row not followed by a common letter differ significantly $(\mathrm{P}<0.05)$.

${ }^{1-2-3}$ Mean values corresponding to the same location and sampling time not followed by a common number differ significantly $(\mathrm{P}<0.05)$ between the batches salted along 3,4 and 5 days.

In the fat of the muscular portion, the peroxide values increased significantly $(\mathrm{P}<0.01)$ during salting and, to a lesser extent, during the post-salting stage. Thus, in the batches salted for 3 or 4 days, the peroxide values decreased significantly $(\mathrm{P}<0.05)$ during the first 14 days of drying-ripening stage and later increased $(\mathrm{P}<0.001)$ until the end of the process; the decrease during the first days of the drying-ripening period may be due the higher degree of peroxide degradation than oxidative phenomena at these stages and in these batches, with a final overall decrease in the peroxide values. In the batches salted for 5 days, the peroxide values increased significantly $(\mathrm{P}<0.05)$ during the first 7 days of drying-ripening, and they then remained relatively constant until the end of the manufacturing process.

In the subcutaneous fat, the peroxide values increased significantly $(\mathrm{P}<0.05)$ during salting, remained relatively constant or even decreased during the post-salting stage, and then increased significantly $(\mathrm{P}<0.001)$ during the drying-ripening period. The latter increase, although also significant $(\mathrm{P}<0.05)$, was less marked in the batches salted for 3 days. 
At almost all the sampling points, and in both the muscular and the subcutaneous fat, the peroxide values were significantly $(\mathrm{P}<0.001)$ higher in the batches salted for 5 days than in those salted for 4 days; the values were also significantly $(\mathrm{P}<0.001)$ higher in batches salted for 4 days than in those salted for 3 days. These data clearly indicate the pro-oxidant effect of the salt. For all sampling times and salting durations, the peroxide values were significantly $(\mathrm{P}<0.05)$ lower in the subcutaneous fat than in the muscular fat, possibly because salt may not reach this area of the pieces, and possibly also because of the lower diffusion in the oxygen.

The final average values were $7.68,17.79$ and 21.72 meq. of $\mathrm{O}_{2} / \mathrm{kg}$ of subcutaneous fat and 10.78, 24.96 and 26.48 meq. of $\mathrm{O}_{2} / \mathrm{kg}$ of muscular fat, in the batches salted for 3, 4 and 5 days, respectively.

The final peroxide values in the fat of the muscular portion were similar to those described by Marra et al. (1999) and by Rodríguez et al. (2001) for raw-cured lacón at the end of the manufacturing process. However, these values were generally higher than those reported for Serrano hams (Flores et al., 1985; Astiasarán et al., 1988) and Iberian hams (Antequera et al., 1992) after a similar ripening time; the final average values in the subcutaneous fat were also higher than those described by Flores et al. (1985) for ham. This may be related to the smaller, thinner lacón pieces, resulting in a larger surface area per unit of weight and, therefore, a larger surface area in contact with the air.

Tables 3,4 and 5 show the variation in the total fatty acid content of the muscular fat throughout the manufacture of the lacón batches salted for 3,4 and 5 days, respectively.

Table 3. Evolution of the total fatty acid content (expressed as \%) in the muscular fat during the manufacture of dry-cured lacón salted along three days (mean \pm standard deviation values of two batches)

\begin{tabular}{|c|c|c|c|c|c|c|c|c|c|}
\hline \multirow[b]{2}{*}{ Fatty acids } & \multirow[b]{2}{*}{ Fresh piece } & \multirow[b]{2}{*}{ After salting } & \multicolumn{2}{|c|}{ Post-salting (days) } & \multicolumn{5}{|c|}{ Drying-ripening (days) } \\
\hline & & & 7 & 14 & 7 & 14 & 28 & 56 & 84 \\
\hline $\mathrm{C14}$ & & & $1.21+0.10$ & & $3 \pm 0.26^{\mathrm{a} 1}$ & & & & $1.13 \pm 0.12^{\mathrm{a} 1}$ \\
\hline C14:1 & $0.01 \pm 0.01^{\mathrm{a} 1}$ & $0.02 \pm 0.01^{\mathrm{a}^{1}}$ & $0.01 \pm 0.01^{\mathrm{a}^{1}}$ & $0.01 \pm 0.01^{\mathrm{a} 1}$ & $0.01 \pm 0.01^{\mathrm{a} 1}$ & ND & $0.01 \pm 0.01^{\mathrm{a} 1}$ & $0.02 \pm 0.01^{\mathrm{a} 1}$ & $0.01 \pm 0.01^{\mathrm{a}^{1}}$ \\
\hline $\mathrm{C} 15$ & $0.08 \pm$ & $0.07 \pm 0$ & $0.06 \pm 0$ & $0.05 \pm 0.00^{12}$ & $.06 \pm 0.01^{\mathrm{al}}$ & $0.06 \pm 0.01^{\mathrm{a} 1}$ & $0.06 \pm 0.01^{\mathrm{a} 1}$ & $0.05 \pm 0.01^{\mathrm{a} 1}$ & $0.04 \pm 0.01^{\mathrm{a} 1}$ \\
\hline C15:1 & $0.03 \pm 0.01^{\mathrm{a}^{1}}$ & $0.02 \pm 0.02^{\mathrm{a}^{1}}$ & $0.04 \pm 0.03^{\mathrm{a} 1}$ & ND & $0.02 \pm 0.02^{\mathrm{a}^{1}}$ & ND & $0.03 \pm 0.02^{\mathrm{a}^{1}}$ & ND & $0.02 \pm 0.01^{\mathrm{a} 1}$ \\
\hline C16 & $22.83 \pm 0.01^{\mathrm{a} 1}$ & $22.23 \pm 0$ & $23.34 \pm 1.02^{\mathrm{a} 1}$ & $23.82 \pm 0.3$ & $22.22 \pm 2.72^{\mathrm{a}^{1}}$ & $22.75 \pm 0.54^{\mathrm{a}^{1}}$ & $21.71 \pm 3.53^{\mathrm{a}^{1}}$ & $22.46 \pm 1.05^{\mathrm{a}^{1}}$ & $21.76 \pm 1.61^{\mathrm{a} 1}$ \\
\hline C16:1 & $0.04 \pm 0$. & $0.04 \pm 0.01^{\mathrm{a} 1}$ & $0.06 \pm$ & 04 & $01 \pm 0.01^{\mathrm{b} 1}$ & $0.03 \pm 0$. & & $0.02=$ & $=0.01^{\mathrm{bl}}$ \\
\hline $\mathrm{C} 17$ & & $0.40 \pm 0.04^{\mathrm{b} 1}$ & & & & $0.34 \pm 0.02^{\mathrm{c} 1}$ & $0.37 \pm 0.08^{\mathrm{bc} 1}$ & $0.26 \pm 0.02^{\mathrm{d} 1}$ & $0.32 \pm 0.06^{\mathrm{bcl}}$ \\
\hline C17:1 & $0.54 \pm 0.01^{\mathrm{al}}$ & $0.41 \pm 0.02^{\mathrm{b} 1}$ & $0.37 \pm 0.10^{\mathrm{b} 1}$ & $0.33 \pm 0.01^{\mathrm{b} 1}$ & $.32 \pm 0.03^{\mathrm{b} 1}$ & $.33 \pm 0.01^{\mathrm{b} 1}$ & $0.32 \pm 0.03^{\mathrm{b} 1}$ & $0.32 \pm 0.02^{\mathrm{b} 1}$ & $0.28 \pm 0.06^{\mathrm{b} 1}$ \\
\hline C18 & $11.63 \pm 0.13^{\mathrm{a} 1}$ & $10.47 \pm 1$ & $12.58 \pm 0.25^{\mathrm{a} 1}$ & $11.19 \pm 0.16^{\mathrm{a} 1}$ & & & & $10.61 \pm 0.22^{\mathrm{a} 1}$ & $10.66 \pm 0.57^{\mathrm{a} 1}$ \\
\hline & $43.15 \pm 0.08^{\mathrm{a} 1}$ & $44.82 \pm 0.56^{\mathrm{ac} 1}$ & $42.25 \pm 4.04^{\mathrm{a} 1}$ & $49.51 \pm 0.04^{\mathrm{bc} * 1}$ & & & & & \\
\hline C18:2 & $15.09 \pm 0.01^{\mathrm{ac} 1}$ & $14.84 \pm 0.27^{\mathrm{ac} 1}$ & $14.89 \pm 2.20^{\mathrm{ac} 1}$ & $10.69 \pm 0.01^{\mathrm{b} * 1}$ & $15.36 \pm 2.20^{\mathrm{cd} 1}$ & $13.36 \pm 2.85^{\mathrm{cb} 1}$ & $18.30 \pm 1.11^{\mathrm{ad} 1}$ & $11.93 \pm 1.67^{\mathrm{cl}}$ & $13.43 \pm 2.79^{\mathrm{c} 1}$ \\
\hline $\mathrm{C20}$ & $0.10 \pm 0.01^{\mathrm{a} 1}$ & $0.07 \pm 0.01^{\mathrm{a} 1}$ & $0.06 \pm 0.05^{\mathrm{a} 1}$ & & & $0.07 \pm 0.01^{\mathrm{a}^{1}}$ & & $0.06 \pm 0.01^{\mathrm{a} 1}$ & $0.07 \pm 0.02^{\mathrm{a} 1}$ \\
\hline C18:3 & & & & & & & & & \\
\hline C20:1 & $0.90 \pm 0.01^{\mathrm{a} 1}$ & $0.89 \pm 0.14^{\mathrm{a} 1}$ & $0.88 \pm 0.12^{\mathrm{a} 1}$ & $0.81 \pm 0.01^{\mathrm{a} 1}$ & $0.72 \pm 0.20^{\mathrm{a} 1}$ & $0.89 \pm 0.16^{\mathrm{a}}$ & $0.90 \pm 0.07^{\mathrm{a} 1}$ & $0.96 \pm 0.34^{\mathrm{a} 1}$ & $0.90 \pm 0.32^{\mathrm{a} 1}$ \\
\hline $\mathrm{C} 21$ & $0.04 \pm 0.01^{\mathrm{a} 1}$ & $0.05 \pm 0.01^{\mathrm{a} 1}$ & $0.05 \pm 0.01^{\mathrm{a}^{1}}$ & $0.04 \pm 0.01$ & $0.04 \pm 0.01$ & $0.04 \pm 0.01^{\mathrm{a}^{1}}$ & $0.04 \pm 0.01^{\mathrm{a} 1}$ & $0.07 \pm 0.03^{\mathrm{a} 1}$ & $0.05 \pm 0.03^{\mathrm{a} 1}$ \\
\hline $\mathrm{C} 20: 2$ & $0.71 \pm 0.01^{\mathrm{acl}}$ & & & $0.43 \pm 0.01^{\mathrm{bc} 1}$ & $0.71 \pm 0.12^{\mathrm{ac} 1}$ & & & $0.60 \pm 0.07^{\mathrm{a} 1}$ & $0.78 \pm 0.19^{\mathrm{a} 1}$ \\
\hline C20:3 & $0.24 \pm 0.01^{\mathrm{a} 1}$ & $0.17 \pm 0.01^{\mathrm{bc} 1}$ & $0.21 \pm 0.02^{\mathrm{a} b 1}$ & $0.11 \pm 0.01^{\mathrm{c} 1}$ & $0.17 \pm 0.0^{\mathrm{bc} 1}$ & $0.16 \pm 0.02^{\text {bc } 1}$ & $0.20 \pm 0.04^{\mathrm{ab} 1}$ & $0.13 \pm 0.01^{\mathrm{bcl}}$ & $0.15 \pm 0.02^{\mathrm{bc} * 1}$ \\
\hline $\mathrm{C} 22$ & $01^{\mathrm{a} 1}$ & 0.61 & $2 \pm 0.46^{\mathrm{a} 1}$ & 0.4 & & $0.56 \pm 0.19^{\mathrm{b} 1}$ & $0.85 \pm 0.06^{\mathrm{ab} 1}$ & $0.48 \pm 0.11^{\mathrm{b} 1}$ & $.12^{\mathrm{bl}}$ \\
\hline C20:4 & $0.14 \pm 0.01^{\mathrm{a} * 1}$ & $0.14 \pm 0.01^{\mathrm{a} 1}$ & $0.13 \pm 0.01^{\mathrm{a} * 1}$ & $0.08 \pm 0.01^{\mathrm{b} 1}$ & $0.13 \pm 0.01^{a * 1}$ & $0.14 \pm 0.01^{\mathrm{bl}}$ & $0.14 \pm 0.03^{\mathrm{ab} * 1}$ & $0.12 \pm 0.01^{\mathrm{ab} 1}$ & $0.15 \pm 0.01^{\mathrm{b} * 1}$ \\
\hline C22:1 & $0.03 \pm 0.01^{\mathrm{a} 1}$ & $0.02 \pm 0.01^{\mathrm{a} 1}$ & $0.03 \pm 0.01^{\mathrm{a} 1}$ & $0.03 \pm 0.01^{\mathrm{a} 1}$ & $0.01 \pm 0.02^{\mathrm{a}^{1}}$ & $0.03 \pm 0.02^{\mathrm{a}^{1}}$ & $0.02 \pm 0.01^{\mathrm{a} 1}$ & $0.02 \pm 0.01^{\mathrm{a} 1}$ & $0.03 \pm 0.01^{\mathrm{a} 1}$ \\
\hline C22:2 & $0.44 \pm 0.01^{\mathrm{a} 1}$ & $1.73 \pm 1.38^{\mathrm{b} 1}$ & $0.62 \pm 0.39^{\mathrm{ab} 1}$ & $0.01 \pm 0.01^{\mathrm{a} 1}$ & $0.56 \pm 0.75^{\mathrm{ab} * 1}$ & $0.43 \pm 0.56^{\mathrm{a} 1}$ & $0.49 \pm 0.64^{\mathrm{a} 1}$ & $0.65 \pm 0.86^{\mathrm{ab} 1}$ & $1.25 \pm 1.41^{\mathrm{a} 1}$ \\
\hline $\mathrm{C} 24$ & $0.26 \pm 0.01^{\mathrm{a} * 1}$ & $0.18 \pm 0.03^{\mathrm{b} * 1}$ & $0.24 \pm 0.03^{\mathrm{a} * 1}$ & $0.13 \pm 0.01^{\mathrm{b} 1}$ & $0.17 \pm 0.01^{\mathrm{b} * 1}$ & $0.18 \pm 0.04^{\mathrm{ac} * 1}$ & $0.21 \pm 0.01^{\mathrm{b} * 1}$ & $0.16 \pm 0.02^{\mathrm{bl}}$ & $0.19 \pm 0.02^{\mathrm{b} 1}$ \\
\hline $\mathbf{S}$ & $37.79 \pm 0.12^{\mathrm{a} 1}$ & $35.33 \pm 0.97^{\mathrm{a}^{1}}$ & $39.00 \pm 2.07^{\mathrm{a}^{1}}$ & $37.25 \pm 0.11^{\mathrm{a} 1}$ & $35.50 \pm 3.96^{\mathrm{a}^{1}}$ & $37.52 \pm 1.31^{\mathrm{a} 1}$ & $35.55 \pm 5.87^{\mathrm{a} 1}$ & $35.39 \pm 1.66^{\mathrm{a} 1}$ & $34.77 \pm 0.96^{\mathrm{a} 1}$ \\
\hline MU & $44.72 \pm 0.07^{\mathrm{ac} 1}$ & $46.21 \pm 0.67^{\mathrm{ac} 1}$ & $43.63 \pm 3.98^{\mathrm{a} 1}$ & $50.73 \pm 0.09^{\mathrm{bc} * 1}$ & $46.64 \pm 0.80^{\mathrm{abc} 1}$ & $46.96 \pm 1.20^{\mathrm{abcl}}$ & $43.79 \pm 4.47^{\mathrm{a} 1}$ & $50.43 \pm 2.53^{\mathrm{bc} 1}$ & $48.63 \pm 3.67^{\mathrm{c} 1}$ \\
\hline $\mathbf{P U}$ & $17.48 \pm 0.03^{\mathrm{ac} 1}$ & $18.44 \pm 1.65^{\mathrm{ac} 1}$ & $17.35 \pm 1.93^{\mathrm{ac} 1}$ & $12.01 \pm 0.05^{\mathrm{b} * 1}$ & $17.86 \pm 3.15^{\mathrm{acl}}$ & $15.52 \pm 2.51^{\mathrm{ab} 1}$ & $20.85 \pm 1.70^{\mathrm{c} 1}$ & $14.17 \pm 0.88^{\mathrm{ab} 1}$ & $16.57 \pm 4.62^{\mathrm{ac} 1}$ \\
\hline $\mathbf{U}$ & $62.21 \pm 0.12^{\mathrm{a} 1}$ & $64.66 \pm 0.98^{\mathrm{a} 1}$ & $61.00 \pm 2.06^{\mathrm{a} 1}$ & $62.75 \pm 0.13^{\mathrm{a} 1}$ & $64.50 \pm 3.94^{\mathrm{a} 1}$ & $62.47 \pm 1.31^{\mathrm{a} 1}$ & $64.65 \pm 6.17^{\mathrm{a} 1}$ & $64.60 \pm 1.66^{\mathrm{a} 1}$ & $65.22 \pm 0.96^{\mathrm{a} 1}$ \\
\hline
\end{tabular}


S: sum of saturated fatty acids; MU: sum of monounsaturated fatty acids; PU: sum of polyunsaturated fatty acids; U: sum of unsaturated fatty acids; ND: Not detected.

${ }^{\mathrm{a}-\mathrm{c}}$ Mean values in the same row (corresponding to the same fatty acid or group of fatty acids) not followed by a common letter differ significantly $(\mathrm{P}<0.05)$.

* The value of this fatty acid or group of fatty acids in this sampling time and salting duration differ significantly $(\mathrm{P}<0.05)$ between the muscular and the subcutaneous fat.

${ }^{1-2-3}$ Mean values corresponding to the same fatty acid (or group of fatty acids) and sampling time not followed by a common number differ significantly $(\mathrm{P}<0.05)$ between the batches salted along 3,4 and 5 days.

Table 4. Evolution of the total fatty acid content (expressed as \%) in the muscular fat during the manufacture of dry-cured lacón salted along four days (mean \pm standard deviation values of two batches)

\begin{tabular}{|c|c|c|c|c|c|c|c|c|c|}
\hline \multirow[b]{2}{*}{ Fatty acids } & \multirow[b]{2}{*}{ Fresh piece } & \multirow[b]{2}{*}{ After salting } & \multicolumn{2}{|c|}{ Post-salting (days) } & \multicolumn{5}{|c|}{ Drying-ripening (days) } \\
\hline & & & 7 & 14 & 7 & 14 & 28 & 56 & 84 \\
\hline C14 & & & $1.45 \pm \mathrm{c}$ & + & $48 \pm 0.12^{\mathrm{a} 1}$ & $1.49 \pm 0.10^{\mathrm{a} 1}$ & $1.67 \pm 0.16^{\mathrm{a} 2}$ & $1.46 \pm 0.03^{\mathrm{a} * 1}$ & $1.53 \pm 0.07^{7 * 1}$ \\
\hline C14:1 & a & mat & $002+001 \mathrm{a} * 1$ & & PO & م02 & $002+00 a_{1}^{a 1}$ & $0.02 \pm 0.01^{\mathrm{al}}$ & $0.02 \pm 0.01^{\mathrm{a} * 1}$ \\
\hline $\mathrm{C} 15$ & & & $0.07 \pm 0.02^{2}$ & & & & $0.08 \pm 0.01^{\mathrm{a} 1}$ & & \\
\hline C15:1 & & & $0.0 / \pm 0$ & & $0.02 \pm 0$ & $0.04 \pm 0.03$ & $0.01 \pm 0.02$ & ND & $0.05 \pm 0.03^{\mathrm{a} 1}$ \\
\hline C16 & $24.69 \pm 0.05^{\text {al }}$ & $25.24 \pm 0.92^{\mathrm{a} * 1}$ & $26.02 \pm 0.17^{\mathrm{a} * 1}$ & $26.43 \pm 0.41^{\mathrm{a} * 1}$ & $26.31 \pm 1.03^{\mathrm{al}}$ & $25.73 \pm 0.21^{a * 1}$ & $27.50 \pm 2.71^{\mathrm{a} 2}$ & $25.98 \pm 0.18^{\mathrm{a} 1}$ & $25.69 \pm 0.12^{\mathrm{a} 1}$ \\
\hline C16:1 & & & $0.03 \pm 0.01^{\mathrm{a} * 1}$ & $0.03 \pm 0.01^{\mathrm{al}}$ & $0.03 \pm 0.01^{\mathrm{a} 1}$ & & & & \\
\hline 17 & & & $.34 \pm 0.01^{\mathrm{al}}$ & $.29 \pm 0.05^{\mathrm{al}}$ & 0.12 & $.06^{\mathrm{a}}$ & $.44 \pm 0.07^{\mathrm{al}}$ & $.46 \pm 0.23^{\mathrm{al}}$ & $0.09^{\mathrm{al}}$ \\
\hline C17:1 & & & $0.40 \pm 0.01$ & al & al & $0.41 \pm 0.05^{\mathrm{al}}$ & al & a1 & $5^{a 1}$ \\
\hline 18 & & & $12.67 \pm 1.69^{\mathrm{abcl}}$ & $13.75 \pm 0.16^{\mathrm{abc} * 2}$ & $14.40 \pm 0.38^{\mathrm{b} 2}$ & $14.48 \pm 0.26^{\mathrm{b} * 1}$ & & $13.05 \pm 0.67^{7 \mathrm{abc} 2}$ & $11.75 \pm 0.63^{\mathrm{cl}}$ \\
\hline C18:1 & $4.96 \pm 0.01^{\mathrm{a} 2}$ & $45.07 \pm 2.38^{\mathrm{a} * 2}$ & $44.24 \pm 1.20^{\mathrm{a} * 1}$ & $45.65 \pm 0.80^{\mathrm{a} 2}$ & $43.53 \pm 0.62^{\mathrm{a}^{2}}$ & $42.34 \pm 1.06^{\mathrm{a} 2}$ & $37.68 \pm 3.51^{\mathrm{b} * 2^{2}}$ & $44.86 \pm 2.23^{\mathrm{a} 2}$ & $44.06 \pm 4.34^{\mathrm{a} 1}$ \\
\hline C18:2 & $12.95 \pm 0.10^{\mathrm{ab} 2}$ & $11.26 \pm 0.88^{\mathrm{ab} 2}$ & $10.94 \pm 1.34^{\mathrm{ab} 2}$ & (1) & $10.36 \pm 0.95^{\mathrm{ab} 2}$ & $11.74 \pm 0.99^{\mathrm{ab} 1}$ & $14.05 \pm 0.15^{\mathrm{b} 2}$ & $10.50 \pm 0.72^{\mathrm{ab} 1}$ & 12.0 \\
\hline 0 & $0.07 \pm 0.01^{\mathrm{al}}$ & $0.23 \pm 0.20^{\mathrm{al}}$ & $0.23 \pm 0.21^{\text {al }}$ & $0.07 \pm 0.01^{\mathrm{al}}$ & $0.06 \pm 0.04^{\mathrm{a} 1}$ & $0.08 \pm 0.01^{\mathrm{al}}$ & $0.08 \pm 0.05^{\mathrm{al}}$ & $0.07 \pm 0.02^{\mathrm{al}}$ & $0.07 \pm 0.06^{\mathrm{al}}$ \\
\hline C18:3 & & & $.09 \pm 0.03$ & $0.00 \pm 0.09^{\circ}$ & & & & $0.72 \pm 0.05^{\mathrm{a} 1}$ & $0.84 \pm 0.18^{\mathrm{a} * 1}$ \\
\hline C20:1 & $0.71 \pm 0.05^{\mathrm{a} 1}$ & $0.87 \pm 0.10^{\mathrm{a} 1}$ & $0.92 \pm 0.03^{\mathrm{a} 1}$ & $0.98 \pm 0.12^{\mathrm{a} 1}$ & $1.10 \pm 0.09^{\mathrm{a} 1}$ & $1.00 \pm 0.22^{\mathrm{a} 1}$ & $0.97 \pm 0.04^{\mathrm{a} 1}$ & $0.88 \pm 0.01^{\mathrm{a} 1}$ & $0.96 \pm 0.05^{\mathrm{a} 1}$ \\
\hline 192 & $0.06 \pm 0.01^{\mathrm{a} 1}$ & $0.05 \pm 0.01^{\mathrm{a} 1}$ & $0.06 \pm 0.01^{\mathrm{a} 1}$ & $0.05 \pm 0.01^{\mathrm{a} 1}$ & $0.06 \pm 0.01^{\mathrm{a} 1}$ & $0.05 \pm 0.02^{\mathrm{a} 1}$ & $0.06 \pm 0.01^{\mathrm{a} 1}$ & $0.05 \pm 0.01^{\mathrm{a} 1}$ & $0.06 \pm 0.01^{\mathrm{a} 1}$ \\
\hline C20:2 & NDD & $0.09 \pm 0.02^{\mathrm{al}}$ & $0.05 \pm 0.03^{\mathrm{al}}$ & $0.04 \pm 0.01^{\mathrm{al}}$ & $0.01 \pm 0.01^{\mathrm{al}}$ & $0.01 \pm 0.01^{\mathrm{al}}$ & $0.05 \pm 0.03^{\mathrm{al}}$ & $0.46 \pm 0.02^{\mathrm{a} 1}$ & $0.28 \pm 0.02^{\mathrm{a} 1}$ \\
\hline C20:3 & $0.11 \pm 0.01^{\mathrm{al}}$ & $0.12 \pm 0.01^{\mathrm{ab} 1}$ & $0.12 \pm 0.01^{\mathrm{ab} * 3}$ & $0.12 \pm 0.01^{\mathrm{ab} 1}$ & $0.14 \pm 0.02^{\mathrm{ab} 1}$ & $0.14 \pm 0.01^{\mathrm{ab} * 1}$ & $0.15 \pm 0.01^{\mathrm{ab} 1}$ & $0.12 \pm 0.01^{\mathrm{b} * 2}$ & $0.13 \pm 0.01^{\mathrm{b} * 1}$ \\
\hline $\mathrm{C} 22$ & $0.56 \pm 0.06^{\mathrm{al}}$ & & & $0.48 \pm 0.05^{\mathrm{al}}$ & & & & $0.44 \pm 0.12^{\mathrm{a} 1}$ & $0.76 \pm 0.32^{\mathrm{a} 1}$ \\
\hline C20:4 & $0.11 \pm 0.02^{\mathrm{a} 2}$ & $0.19 \pm 0.09$ & $0.19 \pm 0.11^{\mathrm{b} * * 1}$ & $0.11 \pm 0.01$ & $0.14 \pm 0.01$ & $0.15 \pm 0.03$ & $0.21 \pm 0.03^{\mathrm{c} * 1}$ & $0.11 \pm 0.01^{\mathrm{ab} 1}$ & $0.15 \pm 0.04$ \\
\hline 1 & $0.03 \pm 0.01^{\mathrm{a} 1}$ & $0.06 \pm 0.03^{\mathrm{a} 2}$ & $0.05 \pm 0.04^{\mathrm{al}}$ & $0.03 \pm 0.01^{\mathrm{a} 1}$ & $0.03 \pm 0.01^{\mathrm{a} 1}$ & $0.03 \pm 0.01^{\mathrm{a} 1}$ & $0.03 \pm 0.04^{\mathrm{a} 1}$ & $0.02 \pm 0.02^{\mathrm{a} 1}$ & $0.04 \pm 0.01^{\mathrm{al}}$ \\
\hline C22:2 & $0.01 \pm 0.01^{\mathrm{al}}$ & $0.02 \pm 0.04^{\mathrm{a} 3}$ & $0.03 \pm 0.01^{\mathrm{al}}$ & $0.03 \pm 0.01^{\mathrm{al}}$ & $0.02 \pm 0.02^{\mathrm{a} 1}$ & $0.03 \pm 0.02^{\mathrm{a} 1}$ & $0.03 \pm 0.02^{\mathrm{a} 1}$ & $0.04 \pm 0.59^{\mathrm{a} 1}$ & $0.04 \pm 0.35^{\mathrm{a} 1}$ \\
\hline $\mathrm{C} 24$ & $0.14 \pm 0.02^{\mathrm{a} 2}$ & $0.24 \pm 0.07^{\mathrm{a} * 1}$ & $0.21 \pm 0.11^{\mathrm{a} * 1}$ & $0.14 \pm 0.01^{\mathrm{al}}$ & $0.17 \pm 0.01^{\mathrm{a} * 1}$ & $0.19 \pm 0.01^{\mathrm{a} * 1}$ & $0.25 \pm 0.05^{\mathrm{a} * 1}$ & $0.13 \pm 0.01^{\mathrm{a} 1}$ & $0.19 \pm 0.04^{\mathrm{al}}$ \\
\hline $\mathbf{S}$ & $39.58 \pm 0.21^{\mathrm{ac} 1}$ & $40.96 \pm 1.34^{\mathrm{ac} * 2^{2}}$ & $42.21 \pm 0.42^{\mathrm{ac} * *^{2}}$ & $42.70 \pm 0.27^{\mathrm{ac} 2}$ & $43.51 \pm 0.67^{\mathrm{ac} 2}$ & $43.23 \pm 0$. & $45.28 \pm 3$ & $0.69^{\mathrm{abc} * 2}$ & $40.58 \pm 1.24^{4^{2}}$ \\
\hline MU & $46.16 \pm 0.25^{\mathrm{al}}$ & $46.58 \pm 2.27^{\mathrm{a} * 1}$ & $45.69 \pm 1.01^{\mathrm{a} * 2^{2}}$ & $47.02 \pm 0.87^{\mathrm{a} 3}$ & $45.08 \pm 0.42^{\mathrm{a}^{2}}$ & $43.84 \pm 1.31^{\mathrm{b} * 2}$ & $39.15 \pm 3.55^{\mathrm{c} * 3}$ & $46.26 \pm 2.10^{\mathrm{ab} 2}$ & $45.62 \pm 4.29^{\mathrm{ab} 2}$ \\
\hline$r 0$ & $14.24 \pm 0.12^{\mathrm{ab} 1}$ & $12.44 \pm 0.92^{\mathrm{ab} 2}$ & $12.08 \pm 1.43^{\mathrm{ab} 2}$ & $10.25 \pm 1.14^{\mathrm{a} 3}$ & $11.38 \pm 1.09^{\mathrm{ab} 2}$ & $12.90 \pm 1.05^{\mathrm{ab} 1}$ & $15.50 \pm 0.13^{\mathrm{b} 2}$ & $11.99 \pm 1.41^{\mathrm{ab} * 1}$ & $13.78 \pm 3.04^{\mathrm{ab} 1}$ \\
\hline $\mathbf{U}$ & $60.42 \pm 0.48^{\mathrm{ac} 1}$ & $59.04 \pm 1.34^{\mathrm{abc} * 2}$ & $57.79 \pm 0.42^{\mathrm{abc} * 2}$ & $57.30 \pm 0.27^{\mathrm{abc} 2}$ & $56.49 \pm 0.67^{\mathrm{abc} 3}$ & $56.77 \pm 0.26^{\mathrm{abc} * 2}$ & $54.72 \pm 3.40^{\mathrm{b} * 2}$ & $58.27 \pm 0.69^{\mathrm{abc} 2}$ & $59.42 \pm 1.24^{\mathrm{c} 2}$ \\
\hline
\end{tabular}

S: sum of saturated fatty acids; MU: sum of monounsaturated fatty acids; PU: sum of polyunsaturated fatty acids; U: sum of unsaturated fatty acids; ND: Not detected.

${ }^{\mathrm{a}-\mathrm{c}}$ Mean values in the same row (corresponding to the same fatty acid or group of fatty acids) not followed by a common letter differ significantly $(\mathrm{P}<0.05)$.

* The value of this fatty acid in this sampling time and salting duration differ significantly $(\mathrm{P}<0.05)$ between the muscular and the subcutaneous fat.

${ }^{1-2-3}$ Mean values corresponding to the same fatty acid (or group of fatty acids) and sampling time not followed by a common number differ significantly $(\mathrm{P}<0.05)$ between the batches salted along 3,4 and 5 days. 
Table 5. Evolution of the total fatty acid content (expressed as \%) in the muscular fat during the manufacture of dry-cured lacón salted along five days (mean \pm standard deviation values of two batches)

\begin{tabular}{|c|c|c|c|c|c|c|c|c|c|}
\hline \multirow[b]{2}{*}{ Fatty acids } & \multirow[b]{2}{*}{ Fresh piece } & \multirow[b]{2}{*}{ After salting } & \multicolumn{2}{|c|}{ Post-salting (days) } & \multicolumn{5}{|c|}{ Drying-ripening (days) } \\
\hline & & & 7 & 14 & 7 & 14 & 28 & 56 & 84 \\
\hline C14 & $1.31 \pm 0.03^{\mathrm{al}}$ & $1.22 \pm 0.09^{\mathrm{al}}$ & $1.25 \pm 0.12^{\mathrm{a} 1}$ & $32 \pm 0.01^{\mathrm{al}}$ & $1.26 \pm 0.90^{\mathrm{al}}$ & $1.36 \pm 0.22^{\mathrm{al}}$ & $1.21 \pm 0.03^{\mathrm{al}}$ & $1.29 \pm 0.19^{\mathrm{al}}$ & $1.13 \pm 0.01^{\mathrm{a} 1}$ \\
\hline C14:1 & $0.02 \pm 0.01 \mathrm{a}^{1}$ & $0.01 \pm 0.01^{\mathrm{al}}$ & $0.02 \pm 0.01^{\mathrm{a} 1}$ & $0.02 \pm 0.01^{\mathrm{al}}$ & $0.02 \pm 0.01^{\mathrm{al}}$ & $0.01 \pm 0.01^{\mathrm{a}^{\mathrm{l}}}$ & $0.01 \pm 0.01^{\mathrm{a} 1}$ & $0.02 \pm 0.01^{\mathrm{a}^{1}}$ & $0.01 \pm 0.01^{\mathrm{a} 1}$ \\
\hline C15 & $0.05 \pm 0.01^{\mathrm{al}}$ & $0.04 \pm 0.01^{\mathrm{a} 1}$ & $0.06 \pm 0.01^{\mathrm{a}^{1}}$ & $0.07 \pm 0.01^{\mathrm{al}}$ & $0.03 \pm 0.01^{\mathrm{a}^{1}}$ & $0.06 \pm 0.01^{\mathrm{a}^{1}}$ & $0.06 \pm 0.02^{\mathrm{a}^{1}}$ & $0.05 \pm 0.01^{\mathrm{a} 1}$ & $0.05 \pm 0.03^{\mathrm{al}}$ \\
\hline C15:1 & $0.06 \pm 0.01^{\mathrm{a} 1}$ & $0.05 \pm 0.02^{\mathrm{a} 1}$ & $0.03 \pm 0.01^{\mathrm{al}}$ & $0.03 \pm 0.01^{\mathrm{al}}$ & $0.04 \pm 0.0^{\mathrm{a} 1}$ & ND & $0.03 \pm 0.01^{\mathrm{al}}$ & $0.03 \pm 0.01^{\mathrm{al}}$ & $0.02 \pm 0.01^{\mathrm{al}}$ \\
\hline C16 & $23.61 \pm 0.64^{4^{\mathrm{b} 1}}$ & ${ }^{1} 23.17 \pm 0.65^{\text {abl }}$ & $23.04 \pm 0.26^{\mathrm{ab} 1}$ & $23.54 \pm 0.01^{\mathrm{ab} 1}$ & $25.91 \pm 1.80^{\mathrm{al}}$ & $23.99 \pm 0.57^{\mathrm{ab} 1}$ & $22.47 \pm 1.65^{\mathrm{bl}}$ & $23.09 \pm 2.35^{\mathrm{ab} 1}$ & $23.56 \pm 0.71^{\mathrm{ab} 1}$ \\
\hline C16:1 & $0.08 \pm 0.02^{\mathrm{a} 1}$ & $0.05 \pm 0.02^{\mathrm{a} 1}$ & $0.03 \pm 0.01^{\mathrm{b} 1}$ & $0.03 \pm 0.01^{\mathrm{b} 1}$ & $0.02 \pm 0.01^{\mathrm{b} 1}$ & $0.02 \pm 0.01^{\mathrm{b} 1}$ & $0.02 \pm 0.01^{\mathrm{bl}}$ & $0.02 \pm 0.01^{\mathrm{bl}}$ & $0.04 \pm 0.02^{\mathrm{ab} 1}$ \\
\hline C17 & $0.38 \pm 0.02^{\mathrm{a} 1}$ & $0.33 \pm 0.02^{\mathrm{a} 1}$ & $0.35 \pm 0.05^{\mathrm{a} 1}$ & $0.40 \pm 0.01^{\mathrm{al}}$ & $0.26 \pm 0.04^{\mathrm{bl}}$ & $0.30 \pm 0.01^{\mathrm{ab} 1}$ & $0.43 \pm 0.04^{\mathrm{a} 1}$ & $0.30 \pm 0.09^{\mathrm{b} 1}$ & $0.32 \pm 0.13^{\mathrm{b} 1}$ \\
\hline C17:1 & $0.40 \pm 0.01^{\mathrm{a} 1}$ & $0.34 \pm 0.01^{\mathrm{b} 1}$ & $0.36 \pm 0.01^{\mathrm{b} 1}$ & $0.44 \pm 0.01^{\mathrm{b} 1}$ & $0.30 \pm 0.04^{\mathrm{bl}}$ & $0.35 \pm 0.05^{\mathrm{b} 1}$ & $0.46 \pm 0.03^{\mathrm{a}^{1}}$ & $0.34 \pm 0.06^{\mathrm{b} 1}$ & $0.34 \pm 0.07^{\mathrm{b} 1}$ \\
\hline C18 & $12.20 \pm 0.94^{\mathrm{a} 1}$ & $12.34 \pm 0.18^{\mathrm{a}^{2}}$ & $11.67 \pm 0.51^{\mathrm{a} 1}$ & $11.58 \pm 0.01^{\mathrm{al}}$ & $12.94 \pm 1.36^{a * 2}$ & $11.04 \pm 1.66^{\mathrm{a}^{2}}$ & $11.92 \pm 0.87^{a 1}$ & $11.64 \pm 0.09^{a 1}$ & $12.72 \pm 0.71^{\mathrm{a} 2}$ \\
\hline C18:1 & $45.10 \pm 1.24^{\mathrm{a}^{2}}$ & $47.45 \pm 0.19^{\mathrm{a} 1}$ & $47.24 \pm 0.54^{\mathrm{a} * 2}$ & $47.07 \pm 0.02^{\mathrm{a} 1}$ & $46.90 \pm 2.16^{\mathrm{a} 1}$ & $48.30 \pm 0.21^{1^{1}}$ & $47.33 \pm 0.98^{\mathrm{a}^{1}}$ & $47.97 \pm 0.47^{\mathrm{a} 1}$ & $48.49 \pm 2.57^{\mathrm{a}^{1}}$ \\
\hline C & $12.64 \pm 0.23^{\mathrm{a}^{2}}$ & $11.32 \pm 0.28^{\mathrm{a} 2}$ & $12.25 \pm 0.55^{\mathrm{al}}$ & $12.04 \pm 0.01^{\mathrm{a} 2}$ & $9.29 \pm 1.03^{\mathrm{a} * 2}$ & $11.35 \pm 0.64^{a 1}$ & $12.53 \pm 2.96^{\mathrm{a} * 2}$ & $11.61 \pm 1.53^{\mathrm{a} 1}$ & $9.82 \pm 2.79^{a 1}$ \\
\hline $\mathrm{C2O}$ & $0.08 \pm 0.01^{\mathrm{a} * 1}$ & $0.05 \pm 0.01^{\mathrm{al}}$ & $0.07 \pm 0.01^{\mathrm{a} 1}$ & $0.04 \pm 0.04^{\mathrm{a} 1}$ & $0.06 \pm 0.02^{\mathrm{a} 1}$ & $0.03 \pm 0.04^{\mathrm{a} 1}$ & $0.07 \pm 0.02^{\mathrm{a} 1}$ & $0.04 \pm 0.01^{\mathrm{a} 1}$ & $0.07 \pm 0.03^{\mathrm{a} l}$ \\
\hline C18:3 & $0.75 \pm 0.01^{\mathrm{a}^{1}}$ & $0.65 \pm 0.01^{\mathrm{a}^{1}}$ & $0.73 \pm 0.02^{\mathrm{a} 1}$ & $0.77 \pm 0.01^{\mathrm{a} 1}$ & $0.61 \pm 0.09^{\mathrm{a} * 1}$ & $0.71 \pm 0.05^{\mathrm{a} 1}$ & $0.84 \pm 0.24^{\mathrm{a}^{1}}$ & $0.73 \pm 0.12^{\mathrm{a}^{1}}$ & $0.61 \pm 0.18^{\mathrm{a}^{1}}$ \\
\hline C20:1 & $0.86 \pm 0.02^{\mathrm{a} 1}$ & $0.99 \pm 0.10^{\mathrm{a} 1}$ & $0.91 \pm 0.04^{\mathrm{a} 1}$ & $0.77 \pm 0.01^{\mathrm{al}}$ & $0.87 \pm 0.07^{\mathrm{a} * 1}$ & $0.82 \pm 0.15^{\mathrm{a}}$ & $0.82 \pm 0.09^{\mathrm{al}}$ & $0.93 \pm 0.01^{\mathrm{al}}$ & $0.96 \pm 0.08^{\mathrm{al}}$ \\
\hline C21 & $0.04 \pm 0.01^{\mathrm{al}}$ & $0.06 \pm 0.01^{\mathrm{al}}$ & $0.05 \pm 0.01^{\mathrm{al}}$ & $0.05 \pm 0.01^{\mathrm{al}}$ & $0.05 \pm 0.01^{\mathrm{a} 1}$ & $0.04 \pm 0.01^{\mathrm{a} 1}$ & $0.05 \pm 0.01^{\mathrm{a} 1}$ & $0.05 \pm 0.01^{\mathrm{a} 1}$ & $0.06 \pm 0.01^{\mathrm{a} 1}$ \\
\hline C20:2 & $0.50 \pm 0.01^{\mathrm{al}}$ & $0.56 \pm 0.03^{\mathrm{a} 1}$ & $0.53 \pm 0.01^{\mathrm{a} 1}$ & $0.44 \pm 0.01^{\mathrm{a} 1}$ & $0.47 \pm 0.07^{\mathrm{a} * 1}$ & $0.47 \pm 0.03^{\mathrm{a}}$ & $0.54 \pm 0.06^{a * 1}$ & $0.55 \pm 0.06^{\mathrm{a} 1}$ & $0.48 \pm 0.14^{\mathrm{a} * 1}$ \\
\hline C20:3 & $0.17 \pm 0.01^{\mathrm{ab} 1}$ & $0.14 \pm 0.01^{\mathrm{ab} * 2}$ & $0.15 \pm 0.03^{\mathrm{ab} 2}$ & $0.13 \pm 0.01^{\mathrm{ab} 1}$ & $5^{\mathrm{b} 1}$ & $0.12 \pm$ & $0.03^{\mathrm{ab} 1}$ & $01^{\mathrm{ab} 1}$ & $\pm 0.05^{\mathrm{ab} 2}$ \\
\hline $\mathrm{C} 22$ & $1.12 \pm 0.02^{\mathrm{a}^{1}}$ & $0.70 \pm 0.21^{\mathrm{bl}}$ & $0.70 \pm 0.17^{\mathrm{b} 1}$ & $0.65 \pm 0.01^{\mathrm{b} 1}$ & $0.42 \pm 0.23^{\mathrm{b} 1}$ & $0.50 \pm 0.01^{\mathrm{bl}}$ & $0.50 \pm 0.14^{\mathrm{bl}}$ & $0.60 \pm 0.10^{\mathrm{bl}}$ & $0.63 \pm 0.30^{\mathrm{b} 1}$ \\
\hline C20:4 & $0.11 \pm 0.01^{\mathrm{a} * 1}$ & $0.12 \pm 0.01^{\mathrm{ab} 1}$ & $0.12 \pm 0.01^{\mathrm{ab} 1}$ & $0.10 \pm 0.01^{\mathrm{ab} 1}$ & $0.09 \pm 0.01^{\mathrm{a} 1}$ & $0.11 \pm 0.01^{\mathrm{ab} 2}$ & $0.13 \pm 0.01^{\mathrm{bl}}$ & $0.13 \pm 0.01^{\mathrm{ab} 1}$ & $0.11 \pm 0.01^{\mathrm{ab} 1}$ \\
\hline C22:1 & $0.04 \pm 0.01^{\mathrm{a}}$ & $0.02 \pm 0.01^{\mathrm{a} 1}$ & $0.03 \pm 0.01^{\mathrm{a} 1}$ & $0.03 \pm 0.01^{\mathrm{al}}$ & $0.01 \pm 0.02^{\mathrm{a} 1}$ & $0.02 \pm 0.01^{\mathrm{a} 1}$ & $0.04 \pm 0.03^{\mathrm{a}^{1}}$ & $0.02 \pm 0.01^{\mathrm{a} 1}$ & $0.03 \pm 0.01^{\mathrm{a}^{1}}$ \\
\hline C22:2 & $0.13 \pm 0.01^{\mathrm{a} 1}$ & $0.09 \pm 0.02^{\mathrm{a} 2}$ & $0.09 \pm 0.01^{\mathrm{a} 1}$ & $0.21 \pm 0.01^{\mathrm{al}}$ & $0.12 \pm 0.05^{\mathrm{a} 1}$ & $0.14 \pm 0.07^{\mathrm{a}}$ & $0.17 \pm 0.04^{\mathrm{a} 1}$ & $0.16 \pm 0.01^{\mathrm{a} 1}$ & $0.14 \pm 0.02^{\mathrm{a} 1}$ \\
\hline $\mathrm{C} 24$ & $0.25 \pm 0.01^{\mathrm{a} * 1}$ & $0.21 \pm 0.04^{\mathrm{a} * 1}$ & $0.20 \pm 0.02^{a * 1}$ & $0.18 \pm 0.01^{\mathrm{a} * 1}$ & $0.13 \pm 0.07^{\mathrm{b} 1}$ & $0.13 \pm 0.01^{\mathrm{bl}}$ & $0.14 \pm 0.03^{\mathrm{b} 2}$ & $0.17 \pm 0.02^{\mathrm{b} * 1}$ & $0.15 \pm 0.08^{\mathrm{b} 1}$ \\
\hline $\mathrm{s}$ & $39.09 \pm 1.54^{\mathrm{a} 1}$ & $38.13 \pm 0.70^{\mathrm{a} 1}$ & $37.41 \pm 0.01^{\mathrm{al}}$ & $37.86 \pm 0.03^{\mathrm{a} 1}$ & $41.11 \pm 0.89^{\mathrm{a}^{2}}$ & $37.48 \pm 0.81^{a^{1}}$ & $36.87 \pm 2.30^{\mathrm{a} 1}$ & $37.27 \pm 2.24^{\mathrm{a} 1}$ & $38.73 \pm 0.85^{\mathrm{a} 1}$ \\
\hline MU & $46.56 \pm 1.26^{\mathrm{a} 1}$ & $48.93 \pm 0.33^{\mathrm{al}}$ & $48.64 \pm 0.52^{\mathrm{a}^{2}}$ & $48.41 \pm 0.02^{\mathrm{a} 1}$ & $48.15 \pm 2.09^{\mathrm{a} 1}$ & $49.56 \pm 0.09^{a 1}$ & $48.71 \pm 1.00^{\mathrm{a} 2}$ & $49.34 \pm 0.53^{\mathrm{a} 1}$ & $49.92 \pm 2.36^{\mathrm{a} 1}$ \\
\hline $\mathbf{P U}$ & $14.36 \pm 0.27^{\mathrm{a} 1}$ & $12.92 \pm 0.37^{\mathrm{a}^{2}}$ & $13.94 \pm 0.53^{\mathrm{a} 2}$ & $13.72 \pm 0.01^{\mathrm{al}}$ & $10.74 \pm 1.20^{\mathrm{a} 2}$ & $12.96 \pm 0.71^{\mathrm{a} 1}$ & $14.40 \pm 3.31^{a^{2}}$ & $13.37 \pm 1.71^{\mathrm{al}}$ & $11.35 \pm 3.21^{\mathrm{a} 2}$ \\
\hline $\mathbf{U}$ & $60.91 \pm 1.54^{\mathrm{a}^{1}}$ & $61.86 \pm 0.70^{\mathrm{a} 1}$ & $62.59 \pm 0.01^{\mathrm{a}^{\mathrm{1}}}$ & $62.14 \pm 0.03^{\mathrm{a}^{1}}$ & $58.88 \pm 0.84^{a} *^{2}$ & $62.51 \pm 0.81^{1^{1}}$ & $63.12 \pm 2.30^{\mathrm{a} 1}$ & $62.73 \pm 2.24^{\mathrm{a} 1}$ & $61.27 \pm 0.83^{\mathrm{a}^{1}}$ \\
\hline
\end{tabular}

S: sum of saturated fatty acids; MU: sum of monounsaturated fatty acids; PU: sum of polyunsaturated fatty acids; U: sum of unsaturated fatty acids; ND: Not detected

${ }^{\mathrm{a}-\mathrm{b}}$ Mean values in the same row (corresponding to the same fatty acid or group of fatty acids) not followed by a common letter differ significantly $(\mathrm{P}<0.05)$.

* The value of this fatty acid in this sampling time and salting duration differ significantly $(\mathrm{P}<0.05)$ between the muscular and the subcutaneous fat.

${ }^{1-2-3}$ Mean values corresponding to the same fatty acid (or group of fatty acids) and sampling time not followed by a common number differ significantly $(\mathrm{P}<0.05)$ between the batches salted along 3,4 and 5 days.

The fatty acid composition of pig fat is very variable and depends on several factors such as the cut of meat, as well as the race, sex and diet of the animal (Wood et al., 2008). The fatty acid composition of the fat in whole cuts used for manufacturing cured products is of great interest because it influences the salting and dehydration processes (Ventanas, 2001), thus determining the suitability of the raw materials for transformation into cured products. It also plays an important role in the development of important sensory characteristic such as the visual aspect, hardness of the fat and aroma of the manufactured products (Ruiz-Carrascal et al., 2000).

In the fresh pieces under study here, the total fatty acid profiles of the muscular and the subcutaneous fat were very similar. The main fatty acids were oleic and palmitic (together representing around $70 \%$ of the total fatty acids), followed by linoleic, estearic and miristic acids. The fatty acid profile in the fresh lacón pieces is consistent with previous reports for pork shoulder fat (Lorenzo et al., 2003; 2008) and also for fresh cuts used to 
produce ham (Melgar et al., 1990; Cava et al., 1997; García-Regueiro \& Díaz, 1997; Davenel et al., 1999). However, the oleic acid contents (around 45\%) were lower than the very high values reported by Ventanas (2001) for the intramuscular fat of the pieces used to produce Iberian ham (50-58\%), and they were also lower than those reported by Melgar et al. (1990) for fresh pieces of pork from Iberian pigs (53\%) and from Iberian x Duroc pigs $(51 \%)$.

Throughout the manufacturing process, the fatty acid profiles of the pieces were similar to those of the fresh pieces, with very small modifications; this is consistent with previous findings for ham (Buscailhon et al., 1994b; Coutron-Gambotti \& Gandemer, 1999).

The salting duration had significant effects $(\mathrm{P}<0.05)$ on the fatty acid profile of the fat of the muscular portion. These effects were most consistent for the polyunsaturated fatty acid content. The polyunsaturated fatty acid contents were higher in the batches salted for 3 days than in the batches salted for 4 or 5 days, and the differences were significant after salting $(18.44 \%, 12.44 \%$ and $12.92 \%$ respectively), and at all sampling points except after 14, 56 and 84 days of drying-ripening; differences in polyunsaturated fatty acid content between batches salted for 4 and 5 days were only significant in the final product, and the values were higher in the batches salted for 4 days (13.78\% and $11.35 \%$ respectively). These differences were mainly due to the different percentages of linoleic acid, which was the main polyunsaturated fatty acid at all sampling points, comprising between $9 \%$ and $18 \%$ of the total fatty acids.

Information about the effect of the salt content on the fatty acid composition of meat products is very scarce. Coutron-Gambotti et al. (1999) reported that there was little difference in the fatty acid contents of intramuscular lipids of ham in relation to different salting times, and that only the polyunsaturated fatty acid contents of the phospholipid fraction were significantly affected; as in the present study, they were highest in the hams salted for the shortest time.

The lower contents of polyunsaturated fatty acids in the sampling points after salting in the most intensely salted batches appear to be related to the higher intensity of oxidative phenomena in these batches. Salt has a well-known pro-oxidant action (Olson \& Rust, 1973; Chen et al., 1984; Shahidi et al., 1988; Kanner et al., 1991), and polyunsaturated fatty acids are the most susceptible to oxidation.

As a consequence of the high percentages of unsaturated fatty acids in the batches salted for the shortest time (3 days), the saturated fatty acid contents were smaller and the differences in the percentages of these fatty acids were significant at all the sampling times, relative to the batches salted for 4 days.

The fatty acid profile in the muscular fat in the lacón pieces at the end of the manufacturing process is consistent with previous observations (Lorenzo et al., 2003, 2008) and is also similar to that reported by other authors in hams of different origins (Delgado et al., 2002; Gandemer, 2002; Larrea et al., 2007). However, the percentage of linoleic acid was higher in the muscular fat of the lacón pieces in which the percentages were already higher in the fresh pieces. This difference may be associated with factors such as the breed, age and feeding. The lacón pieces used in this study were obtained from carcasses of young animals (carcass weight around $90 \mathrm{~kg}$ ) coming from an industrial crossing that were mainly bred for consumption as fresh meat; the characteristics of this meat are very different from those of the meat used to produce the different ham varieties (other races of pigs, older and heavier animals and possibly fed with different types of foodstuff) (Delgado et al., 2002; Gandemer, 2002; Larrea et al., 2007).

The variation in the total fatty acid content of the subcutaneous fat during the manufacture of the lacón batches salted for 3, 4 and 5 days are shown in Tables 6,7 and 8, respectively. 
Table 6. Evolution of the total fatty acid content (expressed as \%) in the subcutaneous fat during the manufacture of dry-cured lacón salted along three days (mean \pm standard deviation values of two batches)

\begin{tabular}{|c|c|c|c|c|c|c|c|c|c|}
\hline \multirow[b]{2}{*}{ Fatty acids } & \multirow[b]{2}{*}{ Fresh piece } & \multirow[b]{2}{*}{ After salting } & \multicolumn{2}{|c|}{ Post-saling (days) } & \multicolumn{5}{|c|}{ Drying-ripening (days) } \\
\hline & & & 7 & 14 & 7 & 14 & 28 & 56 & 84 \\
\hline C14 & $1.21 \pm 0.09^{\mathrm{a} 1}$ & $1.20 \pm 0.07^{\mathrm{a} 1}$ & $1.22 \pm 0.09^{\mathrm{a} 1}$ & $1.33 \pm 0.07^{\mathrm{a} 1}$ & $1.26 \pm 0.42^{\mathrm{a} 1}$ & $1.29 \pm 0.16^{\mathrm{a} 1}$ & $1.32 \pm 0.12^{\mathrm{a} 1}$ & $1.41 \pm 0.14^{\mathrm{a} 1}$ & $1.24 \pm 0.05^{\mathrm{al}}$ \\
\hline C14:1 & $0.02 \pm 0.01^{\mathrm{al}}$ & $0.01 \pm 0.01^{\mathrm{a} 1}$ & $0.02 \pm 0.01^{\mathrm{al}}$ & $0.02 \pm 0.01^{\mathrm{al}}$ & $0.02 \pm 0.01^{\mathrm{a} 1}$ & $0.02 \pm 0.01^{\mathrm{a} 1}$ & $0.02 \pm 0.01^{\mathrm{al}}$ & $0.02 \pm 0.01^{\mathrm{al}}$ & $0.02 \pm 0.01^{\mathrm{al}}$ \\
\hline C15 & $0.07 \pm 0.01^{\mathrm{a} l}$ & $0.06 \pm 0.01^{\mathrm{a} 1}$ & $0.07 \pm 0.02^{\mathrm{a} 1}$ & $0.07 \pm 0.01^{\mathrm{a} 1}$ & $0.05 \pm 0.01^{\mathrm{a} 1}$ & $0.06 \pm 0.01^{\mathrm{a} 1}$ & $0.06 \pm 0.01^{\mathrm{al}}$ & $0.06 \pm 0.01^{\mathrm{a} 1}$ & $0.07 \pm 0.02^{\mathrm{a} 1}$ \\
\hline C15:1 & ND & $0.01 \pm 0.01^{\mathrm{a} 1}$ & ND & $0.01 \pm 0.01^{\mathrm{al}}$ & ND & ND & $0.01 \pm 0.01^{\mathrm{al}}$ & $0.01 \pm 0.01^{\mathrm{al}}$ & $0.01 \pm 0.01^{\mathrm{al}}$ \\
\hline C16 & $23.32 \pm 1.23^{\mathrm{a} 1}$ & $23.31 \pm 0.15^{\mathrm{al}}$ & $22.83 \pm 1.07^{\mathrm{al}}$ & $22.94 \pm 1.20^{\mathrm{al}}$ & $21.82 \pm 2.60^{\mathrm{al}}$ & $22.56 \pm 0.51^{\mathrm{a} 1}$ & $21.40 \pm 0.83^{\mathrm{a} 1}$ & $22.88 \pm 0.87^{\mathrm{al}}$ & $22.16 \pm 0.58^{\mathrm{al}}$ \\
\hline C16:1 & $0.01 \pm 0.01^{1^{a}}$ & $0.01 \pm 0.01^{\mathrm{a} 1}$ & $0.02 \pm 0.01^{\mathrm{al}}$ & $0.02 \pm 0.01^{\mathrm{a} 1}$ & $0.02 \pm 0.01^{\mathrm{a}^{\mathrm{l}}}$ & $0.01 \pm 0.01^{\mathrm{al}}$ & $0.02 \pm 0.01^{\mathrm{al}}$ & $0.02 \pm 0.01^{\mathrm{al}}$ & $0.02 \pm 0.01^{\mathrm{al}}$ \\
\hline $\mathrm{C} 17$ & $0.43 \pm 0.12^{\mathrm{a} 1}$ & $0.38 \pm 0.03^{\mathrm{ab} 1}$ & $0.38 \pm 0.07^{\mathrm{ab} 1}$ & $0.39 \pm 0.09^{\mathrm{ab} 1}$ & $0.25 \pm 0.05^{\mathrm{b} 1}$ & $0.34 \pm 0.01^{\mathrm{ab} 1}$ & $0.30 \pm 0.06^{\mathrm{b} 1}$ & $0.28 \pm 0.02^{\mathrm{b} 1}$ & $0.36 \pm 0.12^{\mathrm{ab} 1}$ \\
\hline C17:1 & $0.44 \pm 0.11^{\mathrm{a} 1}$ & $0.40 \pm 0.02^{\mathrm{a} 1}$ & $0.39 \pm 0.07^{\mathrm{a}^{1}}$ & $0.40 \pm 0.06^{\mathrm{a} 1}$ & $0.32 \pm 0.01^{\mathrm{a} 1}$ & $0.39 \pm 0.01^{\mathrm{a} 1}$ & $0.32 \pm 0.01^{\mathrm{a} 1}$ & $0.38 \pm 0.01^{\mathrm{a} 1}$ & $0.39 \pm 0.05^{\mathrm{a} 1}$ \\
\hline C18 & $11.31 \pm 0.03^{\mathrm{a}}$ & $9.71 \pm 0.57^{\mathrm{al}}$ & $10.94 \pm 0.97^{\mathrm{al}}$ & $10.26 \pm 0.41^{\mathrm{a} 1}$ & $8.81 \pm 0.56^{\mathrm{a} 1}$ & $9.21 \pm 4.38^{\mathrm{a} 1}$ & $9.60 \pm 1.18^{\mathrm{a} 1}$ & $9.30 \pm 0.06^{\mathrm{a} 1}$ & $9.81 \pm 1.08^{\mathrm{al}}$ \\
\hline C18:1 & $46.66 \pm 1.34^{\mathrm{ab} 1}$ & $46.66 \pm 0.97^{\mathrm{ab} 1}$ & $45.14 \pm 0.93^{\mathrm{a} b 1}$ & $43.81 \pm 0.26^{\mathrm{a} 1}$ & $46.45 \pm 3.42^{\mathrm{abl}}$ & ${ }^{1} 45.71 \pm 0.01^{\mathrm{abl}}$ & ${ }^{1} 43.47 \pm 1.14^{\mathrm{a} 1}$ & $47.29 \pm 1.44^{\mathrm{b} 1}$ & $46.44 \pm 3.62^{\mathrm{ab} 1}$ \\
\hline C18:2 & $12.81 \pm 1.77^{\mathrm{a} 1}$ & $15.45 \pm 1.06^{\mathrm{ab} 1}$ & $15.27 \pm 2.70^{\mathrm{ab} 1}$ & $16.21 \pm 2.30^{\mathrm{ab} 1}$ & $15.67 \pm 8.70^{\mathrm{abl}}$ & ${ }^{1} 16.00 \pm 2.83^{\mathrm{ab} 1}$ & ${ }^{1} 18.71 \pm 2.66^{b 1}$ & $14.62 \pm 1.71^{\mathrm{ab} 1}$ & $15.43 \pm 3.18^{\mathrm{abl}}$ \\
\hline $\mathrm{C20}$ & $0.06 \pm 0.02^{\mathrm{a}^{1}}$ & $0.02 \pm 0.05^{\mathrm{a} 1}$ & $0.07 \pm 0.02^{\mathrm{a} 1}$ & $0.07 \pm 0.01^{\mathrm{a} 1}$ & $0.03 \pm 0.05^{\mathrm{a}^{1}}$ & $0.06 \pm 0.01^{\mathrm{a} 1}$ & $0.03 \pm 0.05^{\mathrm{a} 1}$ & $0.05 \pm 0.01^{\mathrm{al}}$ & $0.07 \pm 0.02^{\mathrm{a} 1}$ \\
\hline C18:3 & $0.78 \pm 0.07^{\mathrm{a} 1}$ & $0.93 \pm 0.04^{\mathrm{abl}}$ & $0.93 \pm 0.18^{\mathrm{ab} 1}$ & $1.02 \pm 0.12^{\mathrm{ab} 1}$ & $0.79 \pm 0.28^{\mathrm{ab} 1}$ & $1.02 \pm 0.25^{\mathrm{ab} 1}$ & $1.00 \pm 0.09^{\mathrm{b} 1}$ & $0.95 \pm 0.15^{\mathrm{ab} 1}$ & $0.95 \pm 0.18^{\mathrm{ab} 1}$ \\
\hline C20:1 & $1.01 \pm 0.06^{\mathrm{a} 1}$ & $0.83 \pm 0.03^{\mathrm{ab} 1}$ & $0.86 \pm 0.03^{\mathrm{ab} 1}$ & $0.80 \pm 0.02^{\mathrm{ab} 1}$ & $0.65 \pm 0.07^{\mathrm{b} 1}$ & $0.83 \pm 0.13^{\mathrm{ab}}$ & $0.64 \pm 0.10^{\mathrm{b} 1}$ & $0.83 \pm 0.29^{\mathrm{ab} 1}$ & $0.93 \pm 0.15^{\mathrm{a} 1}$ \\
\hline $\mathrm{C} 21$ & $0.05 \pm 0.01^{\mathrm{a} 1}$ & $0.04 \pm 0.01^{\mathrm{a}^{1}}$ & $0.05 \pm 0.01^{\mathrm{a} 1}$ & $0.04 \pm 0.01^{\mathrm{a} 1}$ & $0.04 \pm 0.01^{\mathrm{a}^{1}}$ & $0.05 \pm 0.01^{\mathrm{a} 1}$ & $0.04 \pm 0.01^{\mathrm{a} l}$ & $0.05 \pm 0.02^{\mathrm{a} 1}$ & $0.06 \pm 0.02^{\mathrm{a} 1}$ \\
\hline C20:2 & $0.67 \pm 0.09^{\mathrm{a} 1}$ & $0.67 \pm 0.03^{\mathrm{a} 1}$ & $0.67 \pm 0.03^{\mathrm{a} 1}$ & $0.66 \pm 0.05^{\mathrm{a} 1}$ & $0.61 \pm 0.32^{\mathrm{a} 1}$ & $0.70 \pm 0.03^{\mathrm{a} 1}$ & $0.74 \pm 0.12^{\mathrm{a} 1}$ & $0.64 \pm 0.06^{\mathrm{a} 1}$ & $0.69 \pm 0.02^{\mathrm{a} 1}$ \\
\hline C20:3 & $0.11 \pm 0.04^{\mathrm{a} 1}$ & $0.11 \pm 0.01^{\mathrm{al}}$ & $0.13 \pm 0.03^{\mathrm{a} 1}$ & $0.13 \pm 0.02^{\mathrm{a} 1}$ & $0.09 \pm 0.03^{\mathrm{a} 1}$ & $0.12 \pm 0.01^{\mathrm{al}}$ & $0.12 \pm 0.02^{\mathrm{a} 1}$ & $0.10 \pm 0.01^{\mathrm{a} 1}$ & $0.12 \pm 0.03^{a^{1}}$ \\
\hline $\mathrm{C} 22$ & $0.27 \pm 0.08^{\mathrm{a} 1}$ & $0.14 \pm 0.19^{\mathrm{a} 1}$ & $0.30 \pm 0.11^{\mathrm{a} 1}$ & $0.30 \pm 0.08^{\mathrm{a} 1}$ & $0.25 \pm 0.11^{\mathrm{a} l}$ & $0.32 \pm 0.02^{\mathrm{a} 1}$ & $0.30 \pm 0.07^{\mathrm{al}}$ & $0.26 \pm 0.06^{\mathrm{a} 1}$ & $0.30 \pm 0.10^{\mathrm{a} 1}$ \\
\hline C20:4 & $0.15 \pm 0.01^{\mathrm{acl}}$ & $0.11 \pm 0.05^{\mathrm{ab} 1}$ & $0.15 \pm 0.01^{\mathrm{ac} 1}$ & $0.16 \pm 0.01^{\mathrm{ac} 1}$ & $0.11 \pm 0.03^{\mathrm{bl}}$ & $0.16 \pm 0.01^{\mathrm{cl}}$ & $0.14 \pm 0.03^{\mathrm{ac} 1}$ & $0.14 \pm 0.01^{\mathrm{ac} 1}$ & $0.16 \pm 0.01^{\mathrm{cl}}$ \\
\hline C22:1 & ND & ND & ND & $0.01 \pm 0.1^{\mathrm{ab} 1}$ & $0.02 \pm 0.01^{\mathrm{b} 1}$ & $0.02 \pm 0.01^{\mathrm{bl}}$ & $0.01 \pm 0.01^{\mathrm{b} 1}$ & $\mathrm{ND}$ & $\mathrm{ND}$ \\
\hline C22:2 & $0.43 \pm 0.33^{\mathrm{a} 1}$ & $0.82 \pm 0.99^{\mathrm{a} 1}$ & $0.37 \pm 0.37^{\mathrm{a} 1}$ & $1.18 \pm 0.88^{\mathrm{ab} 1}$ & $2.59 \pm 2.47^{\mathrm{b} 1}$ & $0.96 \pm 0.70^{\mathrm{ab} 1}$ & $1.56 \pm 1.85^{\mathrm{ab} 1}$ & $0.55 \pm 0.61^{\mathrm{a} 1}$ & $0.59 \pm 0.57^{\mathrm{a} 1}$ \\
\hline C24 & $0.10 \pm 0.03^{\mathrm{a} 1}$ & $0.10 \pm 0.02^{\mathrm{a} 1}$ & $0.12 \pm 0.03^{\mathrm{a} 1}$ & $0.12 \pm 0.03^{\mathrm{a} 1}$ & $0.08 \pm 0.01^{\mathrm{a} 1}$ & $0.10 \pm 0.01^{\mathrm{a} 1}$ & $0.10 \pm 0.01^{\mathrm{al}}$ & $0.10 \pm 0.02^{\mathrm{a} 1}$ & $0.12 \pm 0.03^{\mathrm{a} 1}$ \\
\hline $\mathrm{S}$ & $36.83 \pm 1.02^{\mathrm{al}}$ & $33.99 \pm 1.06^{\mathrm{abc} 1}$ & $35.99 \pm 1.69^{\mathrm{ac} 1}$ & $35.54 \pm 1.47^{\mathrm{abcl}}$ & $32.61 \pm 3.36^{\mathrm{bc}}$ & ${ }^{1} 34.00 \pm 3.68^{\mathrm{acl}}$ & ${ }^{1} 33.16 \pm 1.82^{\mathrm{cl}}$ & $34.40 \pm 0.73^{\mathrm{abc} 1}$ & $34.19 \pm 0.85^{\mathrm{abcl}}$ \\
\hline MU & $48.16 \pm 1.30^{\mathrm{a} 1}$ & $50.27 \pm 1.06^{\mathrm{a}^{1}}$ & $46.46 \pm 0.88^{\mathrm{a} b 1}$ & $45.10 \pm 0.16^{\mathrm{abcl}}$ & $47.50 \pm 3.52^{\mathrm{abl}}$ & ${ }^{1} 47.00 \pm 0.08^{\mathrm{ab} 1}$ & ${ }^{1} 45.10 \pm 1.02^{\mathrm{b} 1}$ & $48.57 \pm 1.71^{\mathrm{a} 1}$ & $47.82 \pm 3.73^{\text {abl }}$ \\
\hline PU & $14.99 \pm 2.31^{\mathrm{al}}$ & $18.11 \pm 2.06^{\mathrm{ab} 1}$ & $17.55 \pm 2.57^{\mathrm{ab} 1}$ & $19.38 \pm 1.62^{\mathrm{ab} 1}$ & $19.87 \pm 6.90^{\text {abl }}$ & ${ }^{1} 19.00 \pm 3.76^{\mathrm{ab} 1}$ & ${ }^{1} 22.33 \pm 0.79^{b 1}$ & $17.03 \pm 2.44^{\mathrm{ab} 1}$ & $17.97 \pm 2.87^{\mathrm{ab} 1}$ \\
\hline $\mathbf{U}$ & $63.17 \pm 1.02^{\mathrm{a}^{1}}$ & $66.06 \pm 0.99^{\mathrm{ab} 1}$ & $64.00 \pm 1.69^{\mathrm{a}^{1}}$ & $64.46 \pm 1.47^{\mathrm{b} 1}$ & $67.39 \pm 3.38^{b 1}$ & $66.00 \pm 3.68^{\mathrm{ab} 1}$ & ${ }^{1} 66.84 \pm 1.81^{\mathrm{b} 1}$ & $65.60 \pm 0.73^{\mathrm{ab} 1}$ & $65.81 \pm 0.84^{\mathrm{ab} 1}$ \\
\hline
\end{tabular}

S: sum of saturated fatty acids; MU: sum of monounsaturated fatty acids; PU: sum of polyunsaturated fatty acids; U: sum of unsaturated fatty acids; ND: Not detected.

${ }^{\mathrm{a}-\mathrm{c}}$ Mean values in the same row (corresponding to the same fatty acid or group of fatty acids) not followed by a common letter differ significantly $(\mathrm{P}<0.05)$.

${ }^{1-2-3}$ Mean values corresponding to the same fatty acid (or group of fatty acids) and sampling time not followed by a common number differ significantly $(\mathrm{P}<0.05)$ between the batches salted along 3,4 and 5 days. 
Table 7. Evolution of the total fatty acid content (expressed as \%) in the subcutaneous fat during the manufacture of dry-cured lacón salted along four days (mean \pm standard deviation values of two batches)

\begin{tabular}{|c|c|c|c|c|c|c|c|c|c|}
\hline \multirow[b]{2}{*}{ Fatty acids } & \multirow[b]{2}{*}{ Fresh piece } & \multirow[b]{2}{*}{ After salting } & \multicolumn{2}{|c|}{ Post-salting (days) } & \multicolumn{5}{|c|}{ Drying-ripening (days) } \\
\hline & & & 7 & 14 & 7 & 14 & 28 & 56 & 84 \\
\hline C14 & $1.62 \pm 0.16^{\mathrm{abl}}$ & $1.34 \pm 0.13^{\mathrm{a} 1}$ & $1.63 \pm 0.09^{\mathrm{ab} 1}$ & $1.82 \pm 0.01^{\mathrm{b} 2}$ & $1.57 \pm 0.18^{\mathrm{ab} 1}$ & $1.50 \pm 0.01^{\mathrm{ab} 1}$ & $2.25 \pm 0.96^{\mathrm{c} 2}$ & $2.93 \pm 0.05^{\mathrm{d} 2}$ & $2.91 \pm 0.04^{\mathrm{d} 2}$ \\
\hline C14:1 & $0.02 \pm 0.02^{\text {acd } 1}$ & $0.01 \pm 0.01^{\mathrm{abcd} 1}$ & ND & $0.01 \pm 0.02^{\mathrm{abcd} 1}$ & $0.01 \pm 0.02^{\mathrm{abd} 2}$ & $0.02 \pm 0.01^{\mathrm{cd} 1}$ & $0.03 \pm 0.01^{\mathrm{cl}}$ & $0.01 \pm 0.01^{\mathrm{d} 1}$ & ND \\
\hline $\mathrm{C15}$ & $0.07 \pm 0.01^{\mathrm{a} 1}$ & $0.07 \pm 0.01^{\mathrm{a} 1}$ & $0.06 \pm 0.02^{\mathrm{a} 1}$ & $0.06 \pm 0.02^{\mathrm{a} 1}$ & $0.05 \pm 0.02^{\mathrm{a} 1}$ & $0.07 \pm 0.01^{\mathrm{a} 1}$ & $0.13 \pm 0.09^{\mathrm{a} 1}$ & $0.20 \pm 0.01^{\mathrm{a} 1}$ & $0.16 \pm 0.06^{\mathrm{a} 1}$ \\
\hline C15:1 & $0.03 \pm 0.01^{\mathrm{a} 1}$ & $0.02 \pm 0.01^{\mathrm{a} 1}$ & ND & ND & ND & ND & ND & ND & ND \\
\hline $\mathrm{C16}$ & $20.97 \pm 7.93^{\text {ac } 1}$ & $19.37 \pm 7.56^{\mathrm{a} 1}$ & $20.44 \pm 8.97^{\mathrm{acl}}$ & $18.60 \pm 0.06^{\mathrm{a} 1}$ & $25.50 \pm 1.81^{\mathrm{bc} 1}$ & ${ }^{1} 24.27 \pm 0.07^{\mathrm{cl}}$ & $22.93 \pm 1.81^{\mathrm{abcl}}$ & $21.56 \pm 0.02^{\mathrm{abcl}}$ & $21.43 \pm 0.21^{\mathrm{abcl}}$ \\
\hline C16:1 & $0.18 \pm 0.25^{\mathrm{a} 2}$ & $0.23 \pm 0.32^{\mathrm{a} 2}$ & $0.20 \pm 0.28^{\mathrm{a} 2}$ & $0.01 \pm 0.01^{\mathrm{b} 1}$ & ND & ND & ND & $\mathrm{ND}$ & ND \\
\hline $\mathrm{C17}$ & $0.43 \pm 0.16^{\mathrm{a} 1}$ & $0.46 \pm 0.08^{\mathrm{a} 1}$ & $0.41 \pm 0.26^{\mathrm{a} 1}$ & $0.41 \pm 0.05^{\mathrm{a} 1}$ & $0.31 \pm 0.12^{\mathrm{a} 1}$ & $0.37 \pm 0.04^{\mathrm{a} 1}$ & $0.64 \pm 0.41^{\mathrm{al}}$ & $0.46 \pm 0.66^{\mathrm{a}}$ & ND \\
\hline C17:1 & $0.52 \pm 0.08^{\mathrm{a} 1}$ & $0.48 \pm 0.04^{\mathrm{a} 1}$ & $0.74 \pm 0.58^{\mathrm{a} 1}$ & $0.64 \pm 0.27^{\mathrm{a} 1}$ & $0.42 \pm 0.13^{\mathrm{a} 1}$ & $0.48 \pm 0.04^{\mathrm{a} 1}$ & $0.80 \pm 0.41^{\mathrm{a} 1}$ & $1.04 \pm 0.26^{\mathrm{a}}$ & $0.75 \pm 0.16^{\mathrm{a} 1}$ \\
\hline $\mathrm{C} 18$ & $13.05 \pm 1.33^{\text {acef } 1}$ & $13.39 \pm 1.27^{\mathrm{acfl}}$ & $11.43 \pm 0.23^{\text {acef } 1}$ & $16.93 \pm 1.97^{\mathrm{b} 1}$ & $12.34 \pm 1.04^{\mathrm{af} 2}$ & $11.59 \pm 2.11^{\text {acef2 }}$ & $9.93 \pm 0.22^{\mathrm{cel}}$ & $10.83 \pm 1.50^{\mathrm{e} 1}$ & $12.34 \pm 0.64^{\mathrm{f} 2}$ \\
\hline C18:1 & $46.80 \pm 5.41^{\mathrm{a} 1}$ & $51.69 \pm 3.57^{\mathrm{b} 2}$ & $51.47 \pm 5.36^{\mathrm{b} 2}$ & $46.22 \pm 0.16^{\mathrm{a} 1}$ & $47.01 \pm 0.95^{\mathrm{a} 1}$ & $46.74 \pm 0.97^{\mathrm{a} 1}$ & $43.26 \pm 5.48^{\mathrm{a} 1}$ & $42.86 \pm 0.04^{\mathrm{a} 2}$ & $42.66 \pm 2.33^{\mathrm{a} 1}$ \\
\hline C18:2 & $13.37 \pm 0.23^{\mathrm{abcd} 1}$ & $10.91 \pm 2.41^{\mathrm{a} 2}$ & $11.31 \pm 2.74^{\mathrm{ad} 1}$ & $12.43 \pm 1.86^{\mathrm{acd} 1}$ & $10.32 \pm 1.34^{\mathrm{a} 2}$ & $12.30 \pm 1.45^{\mathrm{acd} 1}$ & $16.74 \pm 4.82^{\mathrm{bcd} 3}$ & $15.87 \pm 0.38^{\mathrm{cd} 1}$ & $15.41 \pm 0.60^{\mathrm{d} 1}$ \\
\hline $\mathrm{C20}$ & $0.70 \pm 0.87^{\mathrm{a} 1}$ & $0.41 \pm 0.49^{\mathrm{a} 1}$ & $0.44 \pm 0.62^{\mathrm{a} 1}$ & $0.46 \pm 0.57^{\mathrm{a} 1}$ & $0.02 \pm 0.02^{\mathrm{a} 1}$ & $0.04 \pm 0.01^{\mathrm{a} 1}$ & $0.03 \pm 0.01^{\mathrm{a} 1}$ & $0.07 \pm 0.06^{\mathrm{a} 1}$ & $0.08 \pm 0.03^{\mathrm{a} 1}$ \\
\hline C18:3 & $1.04 \pm 0.13^{\mathrm{ab} 1}$ & $0.82 \pm 0.22^{\mathrm{a} 1}$ & $1.02 \pm 0.47^{\mathrm{ab} 1}$ & $1.19 \pm 0.46^{\mathrm{ab} 1}$ & $0.78 \pm 0.14^{\mathrm{a} 1}$ & $0.98 \pm 0.15^{\mathrm{ab} 1}$ & $1.32 \pm 0.32^{\mathrm{b} 1}$ & $0.78 \pm 1.07^{\mathrm{a} 1}$ & $1.32 \pm 0.08^{\mathrm{a} 1}$ \\
\hline C20:1 & $0.44 \pm 0.53^{\mathrm{a} 1}$ & $0.46 \pm 0.56^{\mathrm{a} 1}$ & $0.57 \pm 0.68^{\mathrm{a} 1}$ & $0.80 \pm 1.00^{\mathrm{a} 1}$ & $1.05 \pm 0.01^{\mathrm{a} 1}$ & $0.94 \pm 0.15^{\mathrm{a} 1}$ & $1.05 \pm 0.30^{\mathrm{a} 1}$ & $1.40 \pm 0.20^{\mathrm{a} 1}$ & $1.53 \pm 0.02^{\mathrm{a} 1}$ \\
\hline $\mathrm{C} 21$ & $0.05 \pm 0.01^{\mathrm{a} 1}$ & $0.05 \pm 0.01^{\mathrm{a} 1}$ & $0.04 \pm 0.03^{\mathrm{a} 1}$ & $0.07 \pm 0.04^{\mathrm{a} 1}$ & $0.06 \pm 0.01^{\mathrm{a} 1}$ & $0.05 \pm 0.01^{\mathrm{a} 1}$ & $0.06 \pm 0.01^{\mathrm{a} 1}$ & $0.67 \pm 0.83^{\mathrm{a} 1}$ & $0.02 \pm 0.01^{\mathrm{c} 2}$ \\
\hline C20:2 & $0.16 \pm 0.18^{\mathrm{a} 1}$ & $0.14 \pm 0.16^{\mathrm{a} 1}$ & $0.13 \pm 0.18^{\mathrm{a} 1}$ & $0.13 \pm 0.13^{\mathrm{a} 1}$ & $0.01 \pm 0.02^{\mathrm{a} 1}$ & $0.03 \pm 0.01^{\mathrm{a} 1}$ & $0.04 \pm 0.02^{\mathrm{a} 1}$ & $0.07 \pm 0.01^{\mathrm{a} 1}$ & $0.09 \pm 0.01^{\mathrm{a} 1}$ \\
\hline $\mathrm{C20:3}$ & $0.08 \pm 0.07^{\mathrm{ab} 1}$ & $0.06 \pm 0.07^{\mathrm{a} b 1}$ & $0.08 \pm 0.09^{\mathrm{a} 1}$ & $0.08 \pm 0.11^{\mathrm{ab} 1}$ & $0.15 \pm 0.01^{\mathrm{ab} 1}$ & $0.16 \pm 0.01^{\mathrm{ab} 1}$ & $0.18 \pm 0.03^{\mathrm{ab} 1}$ & $0.31 \pm 0.15^{\mathrm{b} 1}$ & $0.42 \pm 0.01^{\mathrm{ab} 1}$ \\
\hline $\mathrm{C22}$ & $0.20 \pm 0.07^{\mathrm{a} 1}$ & $0.17 \pm 0.07^{\mathrm{a} 1}$ & $0.18 \pm 0.01^{\mathrm{a} 1}$ & $0.19 \pm 0.04^{\mathrm{a} 1}$ & $0.22 \pm 0.05^{\mathrm{a} 1}$ & $0.25 \pm 0.01^{\mathrm{a} 1}$ & $0.33 \pm 0.12^{\mathrm{a} 1}$ & $0.24 \pm 0.25^{\mathrm{a} 1}$ & $0.04 \pm 0.02^{\mathrm{a} 1}$ \\
\hline C20:4 & $0.04 \pm 0.06^{\mathrm{ac} 1}$ & $0.03 \pm 0.04^{\mathrm{acl}}$ & $0.03 \pm 0.05^{\mathrm{ac} 1}$ & $0.04 \pm 0.06^{\mathrm{acl}}$ & $0.08 \pm 0.01^{\mathrm{ab} 1}$ & $0.09 \pm 0.01^{\mathrm{ab} 1}$ & $0.12 \pm 0.03^{\mathrm{bl}}$ & $0.07 \pm 0.10^{\mathrm{ab} 1}$ & ND \\
\hline C22:1 & $0.02 \pm 0.01^{\mathrm{a} 1}$ & $0.01 \pm 0.01^{\mathrm{a} 1}$ & $0.01 \pm 0.01^{\mathrm{a} 1}$ & $0.02 \pm 0.02^{\mathrm{a} 1}$ & ND & $0.01 \pm 0.01^{\mathrm{a} 1}$ & $0.02 \pm 0.01^{\mathrm{a} 1}$ & $0.11 \pm 0.13^{\mathrm{a} 1}$ & $0.21 \pm 0.01^{\mathrm{a} 1}$ \\
\hline C22:2 & ND & ND & ND & ND & ND & ND & ND & $0.01 \pm 0.01^{\mathrm{al}}$ & $0.01 \pm 0.02^{\mathrm{a} 1}$ \\
\hline $\mathrm{C24}$ & $0.09 \pm 0.01^{\mathrm{a} 1}$ & $0.11 \pm 0.01^{\mathrm{ab} 1}$ & $0.10 \pm 0.04^{\mathrm{ab} 1}$ & $0.10 \pm 0.01^{\mathrm{ab} 1}$ & $0.09 \pm 0.03^{\mathrm{a} 1}$ & $0.11 \pm 0.01^{\mathrm{ab} 1}$ & $0.13 \pm 0.04^{\mathrm{ab} 1}$ & $0.16 \pm 0.01^{\mathrm{b} 2}$ & $0.16 \pm 0.01^{\mathrm{b} 1}$ \\
\hline $\mathbf{S}$ & $37.17 \pm 5.47^{\mathrm{abcl}}$ & $35.37 \pm 5.66^{\mathrm{acl}}$ & $34.74 \pm 8.40^{\mathrm{acl}}$ & $38.64 \pm 1.35^{\mathrm{abcl}}$ & $40.16 \pm 0.72^{\mathrm{bc} 2}$ & $38.24 \pm 2.00^{\mathrm{cl}}$ & $36.44 \pm 0.55^{\mathrm{cl}}$ & $37.12 \pm 1.52^{\mathrm{cl}}$ & $38.43 \pm 0.34^{\mathrm{cl}}$ \\
\hline MU & $47.83 \pm 4.93^{\text {acde } 1}$ & $52.67 \pm 3.05^{\mathrm{bd} 2}$ & $52.79 \pm 5.27^{\mathrm{b} 2}$ & $47.69 \pm 0.56^{\operatorname{cde} 1}$ & $48.49 \pm 0.81^{\mathrm{de} 1}$ & $48.19 \pm 0.40^{\mathrm{el}}$ & $45.15 \pm 4.69^{\mathrm{e} 1}$ & $45.43 \pm 0.56^{\mathrm{e} 2}$ & $45.15 \pm 0.15^{\mathrm{e} 2}$ \\
\hline PU & $14.70 \pm 0.12^{\text {abd } 1}$ & $11.99 \pm 2.65^{\mathrm{ad} 3}$ & $12.56 \pm 3.26^{\mathrm{abd} 2}$ & $13.89 \pm 2.25^{\mathrm{acd} 2}$ & $11.35 \pm 1.54^{\mathrm{a} 2}$ & $13.57 \pm 1.60^{\mathrm{acd} 2}$ & $18.41 \pm 5.25^{\mathrm{bcd} 1}$ & $17.14 \pm 1.39^{\mathrm{cd} 1}$ & $15.91 \pm 0.62^{\mathrm{d} 1}$ \\
\hline $\mathbf{U}$ & $62.72 \pm 5.32^{\mathrm{ab} 1}$ & $64.89 \pm 6.03^{\mathrm{a} 1}$ & $65.56 \pm 8.82^{\mathrm{a} 1}$ & $61.58 \pm 1.68^{\mathrm{ab} 1}$ & $59.84 \pm 0.72^{\mathrm{b} 2}$ & $61.76 \pm 2.00^{\mathrm{ab} 1}$ & $63.56 \pm 0.55^{\text {ab1 }}$ & $62.57 \pm 1.94^{\mathrm{ab} 1}$ & $61.10 \pm 0.77^{\mathrm{ab} 1}$ \\
\hline
\end{tabular}

S: sum of saturated fatty acids; MU: sum of monounsaturated fatty acids; PU: sum of polyunsaturated fatty acids; U: sum of unsaturated fatty acids; ND: Not detected

${ }^{\mathrm{a}-\mathrm{c}}$ Mean values in the same row (corresponding to the same fatty acid or group of fatty acids) not followed by a common letter differ significantly $(\mathrm{P}<0.05)$.

${ }^{1-2-3}$ Mean values corresponding to the same fatty acid (or group of fatty acids) and sampling time not followed by a common number differ significantly $(\mathrm{P}<0.05)$ between the batches salted along 3,4 and 5 days. 
Table 8 . Evolution of the total fatty acid content (expressed as \%) in the subcutaneous fat during the manufacture of dry-cured lacón salted along five days (mean \pm standard deviation values of two batches)

\begin{tabular}{|c|c|c|c|c|c|c|c|c|c|}
\hline \multirow[b]{2}{*}{ Fatty acids } & \multirow[b]{2}{*}{ Fresh piece } & \multirow[b]{2}{*}{ After salting } & \multicolumn{2}{|c|}{ Post-salting (days) } & \multicolumn{5}{|c|}{ Drying-ripening (days) } \\
\hline & & & 7 & 14 & 7 & 14 & 28 & 56 & 84 \\
\hline C14 & $1.24 \pm 0.01^{\mathrm{a} 1}$ & $1.25 \pm 0.11^{\mathrm{a} 1}$ & $1.30 \pm 0.16^{\mathrm{a}}$ & $1.36 \pm 0.10^{\mathrm{a}^{1}}$ & $1.47 \pm 0.26^{\mathrm{a} 1}$ & $1.43 \pm 0.29^{\mathrm{a} 1}$ & $1.28 \pm 0.09^{\mathrm{a} 1}$ & $1.31 \pm 0.13^{\mathrm{a} 1}$ & $1.25 \pm 0.05^{\mathrm{a} 1}$ \\
\hline C14:1 & $0.02 \pm 0.01^{\mathrm{al}}$ & $0.02 \pm 0.01^{\mathrm{a} 1}$ & $0.02 \pm 0.01^{\mathrm{a} 1}$ & $0.03 \pm 0.01^{\mathrm{al}}$ & $0.03 \pm 0.01^{\mathrm{a} 1}$ & $0.03 \pm 0.01^{\mathrm{a} 1}$ & $0.01 \pm 0.01^{\mathrm{a} 1}$ & $0.01 \pm 0.01^{\mathrm{a} 1}$ & $0.02 \pm 0.01^{\mathrm{a} 1}$ \\
\hline C15 & $0.05 \pm 0.01^{\mathrm{a}^{1}}$ & $0.04 \pm 0.01^{\mathrm{a} 1}$ & $0.07 \pm 0.01^{\mathrm{a} 1}$ & $0.07 \pm 0.02^{a^{1}}$ & $0.05 \pm 0.01^{\mathrm{a} 1}$ & $0.06 \pm 0.01^{\mathrm{a} 1}$ & $0.07 \pm 0.01^{\mathrm{a} 1}$ & $0.05 \pm 0.01^{\mathrm{a} 1}$ & $0.05 \pm 0.01^{\mathrm{a} 1}$ \\
\hline C15:1 & & $0.01 \pm 0.01^{\mathrm{b} 1}$ & ND & $0.01 \pm 0.01^{\mathrm{b} 1}$ & ND & $0.01 \pm 0.01^{\mathrm{bl}}$ & $0.01 \pm 0.01^{\mathrm{b} 1}$ & $0.01 \pm 0.01^{\mathrm{b} 1}$ & $\mathrm{ND}$ \\
\hline C16 & $22.98 \pm 0.12^{\mathrm{a} 1}$ & $23.56 \pm 0.63^{\mathrm{al}}$ & $22.58 \pm 0.38^{\mathrm{al}}$ & ${ }^{1} 24.09 \pm 0.07^{\mathrm{al}}$ & $23.88 \pm 0.29^{\mathrm{al}}$ & $23.32 \pm 0.61^{a 1}$ & $22.29 \pm 1.18^{\mathrm{a} 1}$ & $22.64 \pm 1.49^{\mathrm{al}}$ & $23.66 \pm 0.56^{\mathrm{al}}$ \\
\hline C16:1 & $0.01 \pm 0.01^{\mathrm{a} 1}$ & $0.01 \pm 0.01^{\mathrm{a}^{1}}$ & $0.01 \pm 0.01^{\mathrm{a} 1}$ & $0.01 \pm 0.01^{\mathrm{a}^{1}}$ & $0.02 \pm 0.02^{\mathrm{a} 1}$ & $0.02 \pm 0.01^{\mathrm{a} 1}$ & $0.02 \pm 0.01^{\mathrm{a} 1}$ & $0.02 \pm 0.01^{\mathrm{a} 1}$ & $0.01 \pm 0.01^{\mathrm{a} 1}$ \\
\hline C17 & $0.41 \pm 0.07^{\mathrm{z}^{1}}$ & $0.30 \pm 0.01^{\mathrm{a} 1}$ & $0.36 \pm 0.05^{\mathrm{al}}$ & $0.35 \pm 0.09^{\mathrm{a} 1}$ & $0.25 \pm 0.02^{\mathrm{b} 1}$ & $0.29 \pm 0.02^{\mathrm{ab} 1}$ & $0.39 \pm 0.05^{\mathrm{al}}$ & $0.30 \pm 0.04^{\mathrm{ab} 1}$ & $0.32 \pm 0.07^{\mathrm{ab} 1}$ \\
\hline C17:1 & $0.45 \pm 0.06^{\mathrm{a}^{1}}$ & $0.35 \pm 0.01^{\mathrm{a} 1}$ & $0.45 \pm 0.01^{\mathrm{a} 1}$ & $0.45 \pm 0.08^{\mathrm{a} 1}$ & $0.42 \pm 0.02^{\mathrm{a} 1}$ & $0.40 \pm 0.06^{\mathrm{a} 1}$ & $0.49 \pm 0.01^{\mathrm{a} 1}$ & $0.36 \pm 0.06^{\mathrm{a}}$ & $0.38 \pm 0.06^{\mathrm{a} 1}$ \\
\hline C18 & $11.06 \pm 0.19^{\mathrm{a} 1}$ & $11.54 \pm 0.24^{\mathrm{a} 1}$ & $19.71 \pm 0.47^{a^{1}}$ & $10.43 \pm 0.06^{\mathrm{a} 1}$ & $9.95 \pm 2.11^{\mathrm{a} 1}$ & $9.33 \pm 1.96^{\mathrm{a} 1}$ & $10.41 \pm 0.19^{\mathrm{a} 1}$ & $10.09 \pm 0.09^{\mathrm{al}}$ & $10.67 \pm 0.24^{\mathrm{al}}$ \\
\hline C18:1 & $49.54 \pm 0.23^{\mathrm{a}^{1}}$ & $48.55 \pm 0.04^{\mathrm{a} 1}$ & ${ }^{1} 48.78 \pm 0.38^{\mathrm{a} 1}$ & ${ }^{1} 48.03 \pm 1.80^{\mathrm{a}^{1}}$ & $49.78 \pm 0.27^{\mathrm{a} 1}$ & $49.28 \pm 0.95^{\mathrm{a}}$ & $48.26 \pm 2.02^{\mathrm{a}^{1}}$ & 50.4 & $\pm 1.61^{\mathrm{al}}$ \\
\hline C18:2 & $11.41 \pm 0.19^{\mathrm{a} 1}$ & $11.38 \pm 0.67^{\mathrm{al}}$ & ${ }^{1} 13.38 \pm 0.15^{\mathrm{al}}$ & $12.14 \pm 1.37^{\mathrm{al}}$ & $11.31 \pm 1.77^{\mathrm{a} 2}$ & $12.75 \pm 0.12^{\mathrm{a} 1}$ & $13.37 \pm 2.70^{\mathrm{a} 2}$ & $11.63 \pm 0.70^{\mathrm{al}}$ & $11.10 \pm 1.52^{\mathrm{a}^{2}}$ \\
\hline $\mathrm{C} 20$ & $0.06 \pm 0.01^{\mathrm{a} 1}$ & $0.05 \pm 0.01^{\mathrm{a} 1}$ & $0.06 \pm 0.01^{\mathrm{a} 1}$ & $0.03 \pm 0.04^{\mathrm{a}^{1}}$ & $0.05 \pm 0.01^{\mathrm{a} 1}$ & $0.06 \pm 0.01^{\mathrm{a} 1}$ & $0.07 \pm 0.02^{\mathrm{a} 1}$ & $0.06 \pm 0.01^{\mathrm{a}^{1}}$ & $0.05 \pm 0.01^{\mathrm{a}}$ \\
\hline C18:3 & $0.78 \pm 0.01^{\mathrm{a} 1}$ & $0.76 \pm 0.05^{\mathrm{a} 1}$ & $0.88 \pm 0.06^{\mathrm{a}}$ & $0.92 \pm 0.01^{\mathrm{a}^{1}}$ & $0.94 \pm 0.06^{\mathrm{a} 1}$ & $0.88 \pm 0.03^{\mathrm{a}^{1}}$ & $1.00 \pm 0.32^{\mathrm{a} 1}$ & $0.79 \pm 0.07^{\mathrm{a}^{1}}$ & $0.78 \pm 0.12^{\mathrm{a}^{1}}$ \\
\hline C20:1 & $1.01 \pm 0.11^{\mathrm{ab} 1}$ & $0.94 \pm 0.14^{\mathrm{a} 1}$ & $0.89 \pm 0.03^{\mathrm{a} 1}$ & $0.83 \pm 0.06^{\mathrm{ab} 1}$ & $0.66 \pm 0.14^{\mathrm{b} 1}$ & $0.83 \pm 0.17^{\mathrm{ab} 1}$ & $0.83 \pm 0.16^{\mathrm{ab} 1}$ & $0.93 \pm 0.02^{\mathrm{a} 1}$ & $0.96 \pm 0.01^{\mathrm{a} 1}$ \\
\hline C21 & $0.06 \pm 0.01^{\mathrm{a} 1}$ & $0.05 \pm 0.01^{\mathrm{a} 1}$ & $0.06 \pm 0.01^{\mathrm{al}}$ & $0.05 \pm 0.01^{\mathrm{a}^{1}}$ & $0.05 \pm 0.01^{\mathrm{a} 1}$ & $0.05 \pm 0.01^{\mathrm{a} 1}$ & $0.04 \pm 0.01^{\mathrm{a} 1}$ & $0.06 \pm 0.01^{\mathrm{a} 1}$ & $0.04 \pm 0.01^{\mathrm{a} 1}$ \\
\hline C20:2 & $0.57 \pm 0.02^{\mathrm{a} 1}$ & $0.56 \pm 0.08^{\mathrm{a} 1}$ & $0.58 \pm 0.04^{\mathrm{al}}$ & $0.47 \pm 0.02^{\mathrm{a} 1}$ & $0.49 \pm 0.04^{\mathrm{al}}$ & $0.51 \pm 0.08^{\mathrm{a} 1}$ & $0.60 \pm 0.07^{\mathrm{al}}$ & $0.57 \pm 0.01^{\mathrm{a} 1}$ & $0.54 \pm 0.08^{\mathrm{al}}$ \\
\hline C20:3 & $0.09 \pm 0.01^{\mathrm{a} 1}$ & $0.07 \pm 0.01^{\mathrm{a} 1}$ & $0.12 \pm 0.01^{\mathrm{a} 1}$ & $0.08 \pm 0.01^{\mathrm{a} 1}$ & $0.07 \pm 0.01^{\mathrm{al}}$ & $0.10 \pm 0.01^{\mathrm{a} 1}$ & $0.09 \pm 0.02^{\mathrm{a} 1}$ & $0.08 \pm 0.01^{\mathrm{a}^{1}}$ & $0.08 \pm 0.01^{\mathrm{a} 1}$ \\
\hline $\mathrm{C} 22$ & $0.23 \pm 0.05^{\mathrm{a} 1}$ & $0.21 \pm 0.01^{\mathrm{a} 1}$ & $0.33 \pm 0.07^{\mathrm{a} 1}$ & $0.21 \pm 0.03^{\mathrm{a} 1}$ & $0.21 \pm 0.04^{\mathrm{a} 1}$ & $0.25 \pm 0.03^{\mathrm{a} 1}$ & $0.29 \pm 0.08^{\mathrm{a} 1}$ & $0.22 \pm 0.05^{\mathrm{a} 1}$ & $0.21 \pm 0.06^{\mathrm{a} 1}$ \\
\hline C20:4 & $0.14 \pm 0.01^{\mathrm{a} 1}$ & $0.13 \pm 0.02^{\mathrm{a} 1}$ & $0.14 \pm 0.02^{\mathrm{a} 1}$ & $0.13 \pm 0.03^{\mathrm{a} 1}$ & $0.11 \pm 0.01^{\mathrm{a} 1}$ & $0.13 \pm 0.01^{\mathrm{a} 1}$ & $0.15 \pm 0.02^{\mathrm{a}^{1}}$ & $0.14 \pm 0.01^{\mathrm{a} 1}$ & $0.14 \pm 0.02^{\mathrm{a} 1}$ \\
\hline C22:1 & $0.01 \pm 0.01^{\mathrm{a} 1}$ & ND & $0.02 \pm 0.01^{\mathrm{a} 1}$ & $0.01 \pm 0.01^{\mathrm{a} 1}$ & $0.02 \pm 0.01^{\mathrm{a} 1}$ & $0.02 \pm 0.01^{\mathrm{a} 1}$ & $0.02 \pm 0.01^{\mathrm{a} 1}$ & $0.02 \pm 0.01^{\mathrm{a} 1}$ & $0.02 \pm 0.01^{\mathrm{a} 1}$ \\
\hline C22:2 & $0.11 \pm 0.10^{\mathrm{a} 1}$ & $0.07 \pm 0.07^{\mathrm{a} 1}$ & $0.10 \pm 0.06^{\mathrm{a} 1}$ & $0.13 \pm 0.10^{\mathrm{a} 1}$ & $0.10 \pm 0.03^{\mathrm{a}^{2}}$ & $0.10 \pm 0.09^{\mathrm{a} 1}$ & $0.12 \pm 0.08^{\mathrm{a} 2}$ & $0.14 \pm 0.13^{\mathrm{a} 1}$ & $0.09 \pm 0.01^{\mathrm{d} 1}$ \\
\hline $\mathrm{C} 24$ & $0.11 \pm 0.02^{\mathrm{a} 1}$ & $0.07 \pm 0.02^{\mathrm{al}}$ & $0.13 \pm 0.02^{\mathrm{a} 1}$ & $0.09 \pm 0.02^{\mathrm{al}}$ & $0.07 \pm 0.01^{\mathrm{a} 1}$ & $0.08 \pm 0.01^{\mathrm{a} 1}$ & $0.10 \pm 0.02^{\mathrm{al}}$ & $0.09 \pm 0.01^{\mathrm{a} 1}$ & $0.09 \pm 0.01^{\mathrm{a} 1}$ \\
\hline $\mathbf{S}$ & $36.24 \pm 0.04^{\mathrm{a} 1}$ & $37.10 \pm 1.00^{\mathrm{a} 1}$ & $34.59 \pm 0.10^{\mathrm{a} 1}$ & $36.68 \pm 0.31^{a 1}$ & $36.01 \pm 2.04^{\mathrm{a} 1}$ & $34.89 \pm 1.03^{\mathrm{a} 1}$ & $34.98 \pm 0.87^{a 1}$ & $34.81 \pm 1.42^{\mathrm{a} 1}$ & $36.38 \pm 0.21^{\mathrm{al}}$ \\
\hline MU & $50.63 \pm 0.31^{\mathrm{a} 1}$ & $49.88 \pm 0.11^{\mathrm{a} 1}$ & $50.18 \pm 0.32^{\mathrm{a} 1}$ & $49.39 \pm 1.79^{a^{2}}$ & $50.94 \pm 0.42^{\mathrm{a}^{1}}$ & $50.59 \pm 0.87^{\mathrm{a}}$ & $49.67 \pm 2.16^{\mathrm{a}^{2}}$ & $51.78 \pm 0.51^{\mathrm{a}^{1}}$ & $50.86 \pm 1.54^{\mathrm{a}}$ \\
\hline PU & $13.11 \pm 0.27^{\mathrm{a} 1}$ & $13.01 \pm 0.90^{\mathrm{a} 2}$ & $15.22 \pm 0.22^{\mathrm{a} 1}$ & $13.91 \pm 1.46^{a^{2}}$ & $13.04 \pm 1.58^{\mathrm{a} 2}$ & $14.51 \pm 0.15^{\mathrm{a}^{2}}$ & $5^{\mathrm{a} 1}$ & $13.41=$ & $1.76^{\mathrm{a}^{2}}$ \\
\hline $\mathbf{U}$ & $63.76 \pm 0.05^{\mathrm{a} 1}$ & $62.90 \pm 1.01^{\mathrm{a} 1}$ & $65.40 \pm 0.11^{\mathrm{a}^{1}}$ & $63.32 \pm 0.30^{a 1}$ & $63.99 \pm 2.04^{\mathrm{a}^{2}}$ & $65.10 \pm 1.03^{\mathrm{a}}$ & $65.02 \pm 0.86^{\mathrm{a}^{\mathrm{a}}}$ & $65.19 \pm 1.42^{\mathrm{a} 1}$ & ${ }^{1} 63.62 \pm 0.21^{a^{a}}$ \\
\hline
\end{tabular}

S: sum of saturated fatty acids; MU: sum of monounsaturated fatty acids; PU: sum of polyunsaturated fatty acids; U: sum of unsaturated fatty acids; ND: Not detected.

${ }^{\mathrm{a}-\mathrm{b}}$ Mean values in the same row (corresponding to the same fatty acid or group of fatty acids) not followed by a common letter differ significantly $(\mathrm{P}<0.05)$.

${ }^{1-2-3}$ Mean values corresponding to the same fatty acid (or group of fatty acids) and sampling time not followed by a common number differ significantly $(\mathrm{P}<0.05)$ between the batches salted along 3,4 and 5 days.

We observed smaller variations in the subcutaneous fat than in the muscular fat when quantifying the different fatty acids during manufacture of the batches salted for different lengths of time. Regarding the effect of the salting duration, there were again significant differences in the polyunsaturated fatty acid content, which was higher in the batches salted for 3 days than in those salted for 4 or 5 days. This difference was significant $(\mathrm{P}<$ 0.05 ) at all post salting sampling times, except after 28,56 and 84 days of drying-ripening. Differences in polyunsaturated fatty acid contents in pieces salted for 4 and 5 days were only significant at the two last sampling times, and the contents were highest in the pieces salted for 4 days. The saturated fatty acid content of the subcutaneous fat in batches salted for 3 days was only significantly lower than in the batches salted for 4 or 5 
days on the $7^{\text {th }}$ day of drying-ripening.

The fatty acid profile in the subcutaneous fat in the lacón at the end of the manufacturing period was similar to that described by other authors (Antequera et al., 1994; Cava et al., 1997; Countron-Gambotti \& Gandemer, 1999; Delgado et al., 2002; Larrea et al., 2007) in the subcutaneous fat of hams of different origin.

The unsaturated fatty acid content was generally lower in the muscular than in the subcutaneous fat of the pieces. This difference, which was particularly marked and significant at many sampling points in the pieces from the batches salted for 4 days, may be due to the pro-oxidant effect of the salt, which barely diffuses through the subcutaneous fat tissue (Wood, 1966), thus favouring the degradation, via oxidation, of unsaturated fatty acids.

\section{Conclusions}

Increasing the salting duration significantly increased lipid oxidation in both the muscular and the subcutaneous fat, and it significantly decreased the polyunsaturated fatty acid content (especially the linolenic acid content) during the manufacture of dry-cured lacón. Shorter salting times (3 days) yielded dry-cured lacón with less oxidized fat that was richer in polyunsaturated fatty acids (i.e. healthier fat).

\section{Acknowledgements}

The authors gratefully acknowledge financial assistance from the Xunta de Galicia (The Regional Government) (Projects 38301B98 and PGIDT01PXI38301PR).

The authors also wish to thank Mr. Juan Vidal Lago from Industrias Cárnicas Portalconsa S.L. (Porriño, Pontevedra, Spain) for his helpful collaboration during this study.

\section{References}

Antequera, T., López-Bote, C. J., Córdoba, J. J., García, C., Asensio, M. A., Ventanas, J., ... Díaz, I. (1992). Lipid oxidative changes in the processing of Iberian pig hams. Food Chemistry, 45, 105-110.

Antequera, T., García, C., López, C., Ventanas, J., Asensio, M. A., \& Córdoba, J. J. (1994). Evolution of different physico-chemical parameters during ripening of iberian ham from Iberian $(100 \%)$ and Iberian $\mathrm{x}$ Duroc pigs (50\%). Revista Española de Ciencia y Tecnología de Alimentos, 34, 178-190.

Astiasarán, I., Beriaín, M. J., Melgar, J., Sánchez-Monje, J. M., Villanueva, R., \& Bello, J. (1988). Estudio comparativo de las características de jamones curados de cerdo blanco elaborados con distinta tecnología. Revista de Agroquímica y Tecnología de Alimentos, 28, 519-528.

Astiasarán, I., Cid, C., Melgar, J., \& Bello, J. (1991). Estudio analítico comparativo de dos tipos de jamones curados: de cerdo ibérico y de cerdo blanco. Revista de Agroquímica y Tecnología de Alimentos, 31, 37-45.

Bañón, S., Cayuela, J. M., Granados, M. V., \& Garrido, M. D. (1999). Pre-cured freezing affects proteolysis in dry-cured hams. Meat Science, 51, 11-16. http://dx.doi.org/10.1016/S0309-1740(98)00067-9

Bellatti, M., \& Reverbi, M. (1997). Prosciutto crudo marchiato: influenza del periodo di salagione sulle caratteristiche chimiche, organolettiche e microbiologiche. Industria Conserve, 72, 359-366.

B. O. E. (1977). Orden de 31 de enero de 1977, por la que se establecen métodos oficiales de análisis de aceites y grasas, cereales y derivados, productos lácteos y productos derivados de la uva. BOE no 167 de 14/07/1977. pp. 15800-15808.

Buscailhon, S., Berdagué, J. L., Gandemer, G., Touraille, C., \& Monin, G. (1994a). Effect of initial pH on compositional changes and sensory traits of French dry-cured hams. Journal of Muscle Foods, 5, 257-270.

Buscailhon, S., Gandemer, G., \& Monin, G. (1994b). Time-related changes in intramuscular lipids of French dry-cured ham. Meat Science, 37, 245-255.

Candek-Potokar, M., Monin, G., \& Zlender, B. (2002). Pork quality, processing, and sensory characteristics of dry-cured hams as influenced by Duroc crossing and sex. Journal of Animal Science, 80, 988-996.

Cava, R., Ruíz, J., López-Bote, C., Martín, L., García, C., Ventanas, J., \& Antequera, T. (1997). Influence of finishing diet on fatty acid profiles of intramuscular lipids, triglicerides and phospholipids in muscles of the Iberian pig. Meat Science, 45, 263-270. http://dx.doi.org/10.1016/S0309-1740(96)00102-7

Chen, J., Piva, M., \& Labuza, T. P. (1984). Evaluation of water binding capacity (WBC) of food fiber source. Journal of Food Science, 49, 59-63.

Coutron-Gambotti, C., \& Gandemer, G. (1999). Lipolysis and oxidation in subcutaneous adipose tissue during dry-cured ham processing. Food Chemistry, 64, 95-101. http://dx.doi.org/10.1016/S0308-8146(98)00079-X 
Coutron-Gambotti, C., Gandemer, G., Rousset, S., Maestrini, O., \& Casabianca, F. (1999). Reducing salt content of dry-cured ham: effect on lipid composition and sensory attributes. Food Chemistry, 64, 13-19. http://dx.doi.org/10.1016/S0308-8146(98)00111-3

Davenel, A., Riaublanc, A., Marchal, P., \& Gandemer, G. (1999). Quality of pig adipose tissue: relationship between solid fat content and lipid composition. Meat Science, 51, 73-79. http://dx.doi.org/10.1016/S0309-1740(98)00099-0

Delgado, G. L., Gómez, C. S., Rubio, L. M. S., Capella, V. S., Méndez, M. D., \& Labastida, R. C. (2002). Fatty acid and triglyceride profiles of intramuscular and subcutaneous fat from fresh and dry-cured hams from Hairless Mexican pigs. Meat Science, 61, 61-65. http://dx.doi.org/10.1016/S0309-1740(01)00163-2

Flores, J., Nieto, P., Bermell, S., \& Alberola, J. (1985). Cambios en los lípidos del jamón durante el proceso de curado, lento y rápido, y su relación con la calidad. Revista de Agroquímica y Tecnología de Alimentos, 25, 117-124.

Folch, J., Lees, M., \& Sloane Stanley, G. H. (1957). A simple method for the isolation and purification of total lipids from animal tissues. Journal of Biological Chemistry, 226, 497-509.

Franco, I., Escamilla, M. C., García, J., García Fontán, M. C., \& Carballo, J. (2006). Fatty acid profile of the fat from Celta pig breed fattened using a traditional feed: Effect of the location in the carcass. Journal of Food Composition and Analysis, 19, 792-799. http://dx.doi.org/10.1016/j.jfca.2006.05.005

Gandemer, G. (2002). Lipids in muscles and adipose tissues, changes during processing and sensory properties of meat products. Meat Science, 62, 309-321. http://dx.doi.org/10.1016/S0309-1740(02)00128-6

García-Regueiro, J. A., \& Díaz, I. (1997). Considerations on the implementation of triglyceride analysis in determining feeding for the Iberian pig. Eurocarne, 56, 75-79, 101.

Garrido, R., Domínguez, R., Lorenzo, J. M., Franco, I., \& Carballo, J. (2012). Effect of the length of salting time on the proteolytic changes in dry-cured lacón during ripening and on the sensory characteristics of the final product. Food Control, 25, 789-796. http://dx.doi.org/10.1016/j.foodcont.2011.11.036

Gou, P., Guerrero, L., \& Arnau, J. (1995). Sex and crossbreed effects on the characteristics of dry-cured ham. Meat Science, 40, 21-31. http://dx.doi.org/10.1016/S0309-1740(94)00021-2

Kanner, J., Harel, S., \& Jaffe, R. (1991). Lipid peroxidation of muscle food as affected by NaCl. Journal of Agricultural and Food Chemistry, 39, 1017-1021.

Larrea, V., Pérez-Munuera, I., Hernando, I., Quiles, A., \& Lluch, M. A. (2007). Chemical and structural changes in lipids during the ripening of Teruel dry-cured ham. Food Chemistry, 102, 494-503. http://dx.doi.org/10.1016/j.foodchem.2006.03.035

Lorenzo, J. M., García Fontán, M. C., Cachaldora, A., Franco, I., \& Carballo, J. (2010). Study of the lactic acid bacteria throughout the manufacture of dry-cured lacón (a Spanish traditional meat product). Effect of some additives. Food Microbiology, 27, 229-235. http://dx.doi.org/10.1016/j.fm.2009.10.003

Lorenzo, J. M., García Fontán, M. C, Franco, I., \& Carballo, J. (2007). Microbiological sucession throughout the manufacture process of dry-cured lacón. Effect of the use of additives. Fleischwirtschaft International, 22, 88-92.

Lorenzo, J. M., García Fontán, M. C., Franco, I., \& Carballo, J. (2008). Proteolytic and lipolytic modifications during the manufacture of dry-cured lacón, a Spanish traditional meat product: Effect of some additives. Food Chemistry, 110, 137-149. http://dx.doi.org/10.1016/j.foodchem.2008.02.002

Lorenzo, J. M., García Fontán, M. C., Franco, I., Prieto, B., \& Carballo, J. (2002). Relationship between biochemical characteristics and sensory quality in dry-cured lacón (a traditional meat product) marketed in Galicia (NW of Spain). Suggestions for improvement. Alimentaria, 338, 31-37.

Lorenzo, J. M., García Fontán, M. C, Gómez, M., Fonseca, S., Franco I., \& Carballo, J. (2012). Study of Micrococcaceae and Staphylococcaceae throughout the manufacture of dry-cured lacón (a Spanish traditional meat product) made without or with additives. Journal of Food Research, 1, 200-211. http://dx.doi.org/10.5539/jfr.v1n1p200

Lorenzo, J. M., González, E., Vidal Lago, J. L., Franco, I., \& Carballo, J. (2005). Study of the losses of weight, and of the parameters related to the stability, during the manufacture of dry-cured lacón made with and without additives. Alimentaria, 367, 56-65. 
Lorenzo, J. M., Prieto, B., Carballo, J., \& Franco, I. (2003). Compositional and degradative changes during the manufacture of dry-cured "lacón". Journal of the Science of Food and Agriculture, 83, 593-601. http://dx.doi.org/10.1002/jsfa.1375

Mariscal, C., García-Ruíz, A., Soriano, A., \& Cabezudo, M. D. (2004). Study of the proteolisis and cathepsin D activity of comercial dry-cured Iberian and Serrano hams. Sciences des Aliments, 24, 221-232. http://dx.doi.org/10.3166/sda.24.221-232

Marra, A. I., Salgado, A., Prieto, B., \& Carballo, J. (1999). Biochemical characteristics of dry-cured lacón. Food Chemistry, 67, 33-37. http://dx.doi.org/10.1016/S0308-8146(99)00104-1

Martín, L., Antequera, T., Córdoba, J. J., Timón, M. L., \& Ventanas, J. (1998). Effects of salt and temperature in proteolysis during ripening of Iberian ham. Meat Science, 49, 145-153. http://dx.doi.org/10.1016/S0309-1740(97)00129-0

Melgar, M. J., Sánchez-Monje, J. M., \& Bello, J. (1990). A study of the changes in the chemical properties of fat during ham curing. Grasas y Aceites, 41, 299-306.

Moller, J. K. S., Jakobsen, M., Weber, C. J., Martinussen, T., Skibsted, L. H., \& Bertelsen, G. (2003). Optimisation of colour stability of cured ham during packaging and retail display by a multifactorial design. Meat Science, 63, 169-175. http://dx.doi.org/10.1016/S0309-1740(02)00066-9

Monin, G., Marinova, P., Talmant, A., Martin, J. F., Cornet, M., Lanore, D., \& Grasso, F. (1997). Chemical and structural changes in dry-cures hams (Bayonne Hams) during processing and effects of the dehairing tecnique. Meat Science, 47, 29-47. http://dx.doi.org/10.1016/S0309-1740(97)00038-7

Monin, G., Virgili, R., Cornet, M., Gandemer,G., \& Grasso, F. (1996). Composition chimique et caractéristiques physiques de 6 types de jambons d'Europe latine. Actes du III Symposium International sur le Porc Méditerranéen, Benevento (Italy), 1995, Produzione Animale, Vol. IX, serie III, 219-230.

Motilva, M. J., \& Toldrá, F. (1993). Effect of curing agents and water activity on pork muscle and adipose subcutaneous tissue lipolytic activity. Zeitschrift für Lebensmittel-Untersuchung und-Forschung, 196, 228-232.

Olson, D. G., \& Rust, R. E. (1973). Oxidative rancidity in dry-cured hams: Effect of low pro-oxidant and antioxidant salt formulations. Journal of Food Science, 38, 251-253.

Rico, E., Toldrá, F., \& Flores, J. (1990). Activity of cathepsin D as affected by chemical and physical dry-curing parameters. Zeitschrift für Lebensmittel-Untersuchung und -Forschung, 191, 20-23.

Rodríguez, M. P., Carballo, J., \& López, M. (2001). Characterization of the lipid fraction of some Galician (NW of Spain) traditional meat products. Grasas $y$ Aceites, 52, 291-296. http://dx.doi.org/10.3989/gya.2001.v52.i5.370

Ruiz-Carrascal, J., Ventanas, J., Cava, R., Andrés, A. I., \& García, C. (2000). Texture and appearance of dry-cured ham as affected by fat content and fatty acid composition. Food Research International, 33, 91-95. http://dx.doi.org/10.1016/S0963-9969(99)00153-2

Sárraga, C., Gil, M., Arnau, J., Monfort, J. M., \& Cussó, R. (1989). Effect of curing salt and phosphate on the activity of porcine muscle proteases. Meat Science, 25, 241-249. http://dx.doi.org/10.1016/S0309-1740/89/\$03.50

Schivazappa, C., Virgili, R., Degni, M., \& Cerati, C. (1998). Effetto della tipologia suina di provenienza su alcune caratteristiche del prosciutto di Parma. Industria Conserve, 73, 110-116.

Shahidi, F., Rubin, L. J., \& D'Souza, L. A. (1986). Meat flavor volatiles: A review of the composition, technique of analysis and sensory evaluation. Critical Reviews in Food Science and Nutrition, 24, 141-243. http://dx.doi.org/10.1080/10408398609527435

Shahidi, F., Rubin, L. J., \& Wood, D. F. (1988). Stabilization of meat lipids with nitrite-free curing mixtures. Meat Science, 22, 73-80. http://dx.doi.org/10.1016/S0309-1740/88/\$03.50

Toldrá, F., \& Flores, J. (1998). The role of muscle proteases and lipases in flavour development during the processing of dry-cured ham. Critical Reviews in Food Science and Nutrition, 38, 331-352. http://dx.doi.org/10.1080/10408699891274237

Toldrá, F., \& Flores, M. (2007). Processed pork meat flavors. In Handbook of food product manufacturing, Vol. 2 In Y. H. Hui, R. Chandan, S. Clark, N. Cross, J. Dobbs, W. J. Hurst, L. M. L. Nollet, E. Shimoni, N. Sinha, 
E. B. Smith, S. Surapat, A. Titchenal \& F. Toldrá (Eds.) (pp. 279-299). Hoboken, NJ, USA: John Wiley Interscience of NY.

Toldrá, F., Flores, M., \& Sanz, Y. (1997). Dry-cured ham flavour: enzymatic generation and process influence. Food Chemistry, 59, 523-530. http://dx.doi.org/10.1016/S0308-8146(97)00013-7

Toscani, T., Virgili, R., Corbari, G., \& Calzolari, L. (2000). Effects of different processing plants on proximate composition, proteolysis and hardness of Parma ham. Industria Conserve, 75, 259-270.

Ventanas, J. (2001). Transformaciones de los lípidos. In Tecnología del jamón ibérico In J. Ventanas (Ed.). (pp. 298-309). Madrid, Spain: Editorial Mundi Prensa.

Vilar, I., García Fontán, M. C., Prieto, B., Tornadijo, M. E., \& Carballo, J. (2000). A survey on the microbiological changes during the manufacture of dry-cured lacón, a Spanish traditional meat product. Journal of Applied Microbiology, 89, 1018-1026. http://dx.doi.org/10.1046/j.1365-2672.2000.01210.x

Wood, F. W. (1966). The diffusion of salt in pork muscle and fat tissue. Journal of the Science of Food and Agriculture, 17, 138-140.

Wood, J. D., Enser, M., Fisher, A. V., Nute, G. R., Sheard, P. R., Richardson, R. I., ... Whittington, F. M. (2008). Fat deposition, fatty acid composition and meat quality: A review. Meat Science, 78, 343-358. http://dx.doi.org/10.1016/j.meatsci.2007.07.019

\section{Copyrights}

Copyright for this article is retained by the author(s), with first publication rights granted to the journal.

This is an open-access article distributed under the terms and conditions of the Creative Commons Attribution license (http://creativecommons.org/licenses/by/3.0/). 